\title{
A diffusion-convection problem with drainage arising in the ecology of mangroves
}

\author{
C. J. VAN DUIJN \\ Technische Universiteit Eindhoven, PO Box 513, 5600 Eindhoven, The Netherlands \\ G. GALiAnO \\ Dipartimento de Matemáticas, Universidad de Oviedo, Avda. Calvo Sotelo s/n, 33007 Oviedo, \\ Spain \\ AND \\ M. A. PELETIER ${ }^{\dagger}$ \\ Centrum voor Wiskunde en Informatica, PO Box 94079, 1090 GB Amsterdam, The Netherlands
}

[Received 6 August 1999 and in revised form 3 January 2000]

\begin{abstract}
We consider both stationary and time-dependent versions of a model describing the vertical movement of water and salt in a porous medium in which a continuous extraction of water takes place (by the roots of mangroves). The problem is formulated in terms of a coupled system of partial differential equations for the salt concentration and the water flow which generalizes previous models. We study the existence and uniqueness of solutions and the conditions under which the maximum principle does hold, showing a counter-example for the general situation. We also analyse the stability of the steady state solution. Finally, we investigate the occurrence of dead cores (sets where the threshold salt concentration is attained) by means of the comparison principle in the stationary problem and of suitable energy estimates in the evolution problem.
\end{abstract}

Keywords: Comparison principle, dead cores, existence, parabolic-elliptic PDEs, uniqueness

\section{Introduction}

Mangroves grow on saturated soils or muds which are subject to regular inundation by tidal water with salt concentration $c_{w}$ close to that of sea water see, for example, [9]. The mangrove roots take up fresh water from the saline soil and leave behind most of the salt, resulting in a net flow of water downward from the soil surface, which carries salt with it. As pointed out by Passioura et al. [13], in the absence of lateral flow, the steady state salinity profile in the root zone must be such that the salinity around the roots is higher than $c_{w}$, and that the concentration gradient is large enough so that the advective downward flow of salt is balanced by the diffusive flow of salt back up to the surface. In [13] the authors disregarded daily variations of evapotranspiration, and presented steady state equations governing the flow of salt and uptake of water in the root zone, assuming that there is an upper limit $c_{c}$ to the salt concentration at which roots can take up water, and that the rate of uptake of water is proportional to the difference between the local concentration $c$ and the assumed upper limit $c_{c}$. They also assumed that the root zone is unbounded, and that the constant of proportionality for root water uptake is independent of depth through the soil. They gave numerical

\footnotetext{
${ }^{\dagger}$ Author for correspondence.
} 
results for variation of salt concentration with depth, obtained from a perturbation solution of the steady state water and salt flow equations.

Their results showed salt concentration increasing with depth from the value $c_{w}$ at the surface, and approaching the limiting value $c_{c}$ at large depth, with a corresponding decrease in water uptake from a maximum at the surface to the limiting value of zero at large depth. The assumption of an infinitely deep root zone requires that the concentration at large depth approaches $c_{c}$, otherwise the total uptake would be infinite. For real mangroves the root distribution is not uniform and does not extend to an infinite depth. For one mangrove species Lin and Sternberg [11] measured the root distribution and found that the root density decreased with depth, with more than half of the fine roots being contained in the top $50 \mathrm{~cm}$ of the soil. The depth distribution of root water uptake is expected to be related to the distribution of fine roots in the soil.

In this paper our aim is to extend the steady state model of [13] in two important ways. First, we will consider more general root water uptake functions, which vary with depth and which depend on a general power $p$ of the concentration difference $c_{c}-c$. Denoting by $S$ the volume of water taken up by the roots per unit volume of porous material per unit time, we use

$$
S:= \begin{cases}\kappa(z)\left(1-\frac{c}{c_{c}}\right)^{p} & \text { for } 0 \leqslant c \leqslant c_{c}, \\ 0 & \text { for } c>c_{c},\end{cases}
$$

where $p>0$ and $\kappa(z)$ is determined by the root distribution as a function of the depth $z$ below the soil surface. This root distribution function will be non-negative, and in accordance with [11] we assume that it is non-increasing with $z$. Passioura et al. [13] used the value $p=1$ corresponding to a linear dependence of uptake on concentration difference, which is consistent with the assumption that uptake is governed by osmotic pressure difference. However, there is no experimental evidence for this choice. Therefore, we investigate the consequences of more general values of $p$ in the uptake model. In particular, we show that the behaviour of the salinity profile differs in an essential manner between the two cases $p<1$ and $p \geqslant 1$.

As shown in Remark 2.1, the estimated values of the physical parameters imply a time scale which allows us to disregard daily variations in the salt concentration at the boundary and which yields, well within the life span of the mangroves, a steady configuration in which diffusion balances the tree-induced convection. Therefore, we will study time-dependent behaviour of salt concentration and flow. In addition to the theoretical issues of existence and uniqueness, we rigorously show some characteristic qualitative properties of the solutions. Using a finite difference solution procedure, we also demonstrate these properties numerically.

The outline of the paper is the following: in Section 2 we formulate a mathematical model for arbitrary root distribution $\kappa$. This model involves two coupled differential equations: a convectiondiffusion equation for the transport of salt, and an ordinary differential equation describing the fluid balance. We restrict ourselves to one-dimensional transport only.

The time-independent state is considered in Section 3. Under stationary conditions the model reduces to a single second-order ordinary differential equation, with well known properties of existence, uniqueness and comparison of solutions. We present a qualitative analysis for two simple but realistic functional forms of the root distribution $\kappa$. Both choices allow us to use a phase plane argument to investigate the steady state.

In Section 4 we discuss some results concerning the evolution problem. The existence of solutions is shown by means of a fixed point argument. The uniqueness and comparison of solutions is a more subtle problem. Under certain restrictive conditions uniqueness is proven. We also show 
that a comparison principle holds between spatial monotonic solutions and general solutions. We present a counter-example showing that in general terms a comparison principle cannot be expected.

In Section 5 we prove some qualitative properties of solutions. In particular, we demonstrate the stability of the steady state solution.

Next, in Section 6, we study the formation of dead cores, regions in which the concentration reaches the threshold value $c_{c}$. We first state a result for the stationary problem, giving conditions under which solutions have a dead core. The proof uses the comparison principle. For the timedependent case, such a principle does not hold in general, and here we have to rely on techniques using energy estimates to show the occurrence of dead cores.

Finally, in Section 7, we show some numerical examples.

\section{The mathematical model}

In this section we formulate the mathematical model which describes the salt movement below the surface where the mangroves are growing and the uptake of fresh water by the root system of the mangroves. We consider the case where the mangroves are present in the horizontal $x, y$ plane, with an homogeneous porous medium located below this plane. This porous medium is characterized by a constant porosity $\theta$, indicating that we are assuming the mangroves roots to be homogenized throughout the porous medium, without affecting its properties. As discussed in the introduction, they are accounted for by the distribution function $\kappa$. Assuming further that the hydrodynamic dispersion tensor, $\boldsymbol{D}$, is constant and isotropic, i.e. neglecting the velocity dependence in the mechanical dispersion, we find for the salt concentration the equation, see [4],

$$
\theta \frac{\partial c}{\partial \tau}+\operatorname{div}(c \boldsymbol{q}-\theta \boldsymbol{D} \nabla c)=0,
$$

where the vector $\boldsymbol{q}$ denotes the specific discharge of the fluid, $\boldsymbol{D}=D \boldsymbol{I}, \boldsymbol{I}$ is the identity matrix and $\tau$ denotes time. We also have a fluid balance. Disregarding density variations in the mass balance equation of the fluid, we obtain a fluid volume balance expressed by

$$
\operatorname{div} \boldsymbol{q}+S=0,
$$

where $S$ is given by (1.1). If the mangroves are uniformly distributed throughout the $x, y$-plane and there is no lateral fluid flow, we may consider the problem as one-dimensional in the vertical $z$-direction. If the $z$-axis is positive when pointing downwards, the flow domain is characterized by the interval $0<z<L<\infty$. In the one-dimensional setting equations (2.1) and (2.2) combined with (1.1) become

$$
\theta \frac{\partial c}{\partial \tau}+\frac{\partial c q^{z}}{\partial z}-\theta D \frac{\partial^{2} c}{\partial z^{2}}=0,
$$

with $q^{z}$ the $z$-component of $\boldsymbol{q}$, and

$$
\frac{\partial q^{z}}{\partial z}+\kappa(z)\left(1-\frac{c}{c_{c}}\right)^{p}=0,
$$

which we want to solve for $0<z<L$ and $\tau>0$, say. Note that in writing (2.4) we implicitly assume that $c \leqslant c_{c}$. We prescribe along the bottom of the domain a no-flow condition for water and 
salt:

$$
q^{z}(L, \tau)=\frac{\partial c}{\partial z}(L, \tau)=0 \quad \text { for all } \quad \tau>0 .
$$

Along the top boundary and initially we assume the salt concentration to be given:

$$
c(0, \tau)=c_{D}(\tau) \quad \text { for all } \quad \tau>0,
$$

and

$$
c(z, 0)=c_{0}(z) \quad \text { for all } \quad 0<z<L .
$$

In many cases of practical interest one would choose the seawater salt concentration along the top boundary, implying $c_{D}(\tau)=c_{w}$ for all $\tau>0$. Throughout this work we assume

$$
0 \leqslant c_{0}(z), c_{D}(\tau) \leqslant c_{c} \quad \text { for all } \quad 0 \leqslant z \leqslant L \text { and } \tau \geqslant 0 .
$$

Regarding the root distribution, $\kappa(z)$, we shall keep in mind the following two characteristic examples. For the first, we assume that the function $\kappa$ is a positive constant above a certain depth $z_{*}$, and zero below that depth, i.e.

$$
\kappa(z):= \begin{cases}\kappa_{0} / z_{*} & 0<z<z_{*}, \\ 0 & z_{*}<z<\infty .\end{cases}
$$

For the second distribution we assume that the strength of the uptake decreases with depth according to

$$
\kappa(z):=\kappa_{0} / z_{*}\left(1+z / z_{*}\right)^{-2},
$$

where $z_{*}$ is a reference depth. This choice is inspired by a transformation that allows for a phaseplane analysis of the associated stationary problem (see Section 3). Both distributions have the same weight $\kappa_{0}$ since both of (2.9) and (2.10) satisfy

$$
\int_{0}^{\infty} \kappa(z) \mathrm{d} z=\kappa_{0} .
$$

Therefore for both distributions the quantity $\kappa_{0}$ is the total amount of root water uptake in the profile with no salt present, in volume per unit surface per unit time, i.e. the transpiration rate of the mangrove plants in the absence of salinity. For the first root distribution (2.9) the depth $z_{*}$ is at the bottom of the root zone. For the second distribution (2.10)

$$
\int_{0}^{z *} \kappa(z) \mathrm{d} z=\frac{1}{2} \kappa_{0},
$$

so $z_{*}$ corresponds to the median depth in that case. Note that for the distribution defined by (2.10) a mean depth cannot be defined because the relevant integral diverges. We recast the equations in an appropriate dimensionless form. Introducing the dimensionless variables and constants

$$
\begin{cases}u:=\frac{c}{c_{c}}, & u_{0}:=\frac{c_{0}}{c_{c}}, \quad u_{D}:=\frac{c_{D}}{c_{c}}, \quad x:=\frac{z}{z_{*}}, \quad d:=\frac{L}{z_{*}}, \\ t:=\frac{D \tau}{z_{*}^{2}}, \quad q:=\frac{z_{*} q^{z}}{\theta D}, \quad k:=\frac{z_{*}^{2} \kappa}{\theta D}, \quad k_{0}:=\frac{z_{*} \kappa_{0}}{\theta D},\end{cases}
$$


we arrive at the following mathematical statement. Let $Q_{T}:=\Omega \times(0, T]$ and $\Omega:=(0, d)$, with $d \in(0, \infty)$ given and $T \in(0, \infty)$ arbitrarily chosen. Find $u, q: \bar{Q}_{T} \rightarrow \mathbb{R}$ such that

$$
(\mathrm{P})\left\{\begin{array}{c}
u_{t}+(u q)_{x}-u_{x x}=0 \\
q_{x}+f(x, u)=0 \\
\text { with } \\
u(0, t)=u_{D}(t) \\
u_{x}(d, t)=q(d, t)=0 \\
u(x, 0)=u_{0}(x) \text { for } x \in \Omega .
\end{array} \quad \text { in } Q_{T},\right.
$$

Here the subscripts $t$ and $x$ denote partial differentiation with respect to these variables. Note that (2.8) implies

$$
0 \leqslant u_{D}(t), u_{0}(x) \leqslant 1
$$

for all $x \in(0, d)$ and $t \in(0, T)$. In Problem $\mathrm{P}$ we introduced the notation

$$
f(x, s):=k(x)(1-s)_{+}^{p} .
$$

The two characteristic distributions (2.9) and (2.10) become

$$
k(x):= \begin{cases}k_{0} & 0<x<1, \\ 0 & 1<x<d,\end{cases}
$$

or

$$
k(x):=\frac{k_{0}}{(1+x)^{2}} .
$$

Instead of restricting ourselves to the special case (2.13), with $k$ for example given by (2.14) or (2.15), we will consider in Problem P a function $f: \bar{\Omega} \times[0,1] \rightarrow \mathbb{R}$ satisfying

$$
\text { (H) }\left\{\begin{array}{l}
\text { 1.1. } f(x, \cdot) \in C([0,1]) \quad \text { for a.e. } x \in \Omega ; \\
\text { 1.2. } f(\cdot, s) \in L^{\infty}(\Omega) \quad \text { for all } \quad s \in[0,1] ; \\
\text { 2. } f(x, \cdot) \text { is non-increasing in }[0,1] \quad \text { and } f(x, 1)=0 \text { for a.e. } x \in \Omega .
\end{array}\right.
$$

Note that $\left(H_{2}\right)$ implies $f \geqslant 0$ in $\bar{\Omega} \times[0,1]$.

REMARK 2.1 Using [13] as a reference we find the following values for the physical constants: $D=7 \cdot 10^{-5} \mathrm{~m}^{2} /$ day, $\theta=0.5$, and $\kappa_{0} / z^{*}=0.1 /$ day. Taking $z^{*}$ in the range $0.2-0.5 \mathrm{~m}$, this implies a time scale in the range $2-10$ years.

\section{The stationary problem}

To select candidates for the long-term behaviour of time-dependent solutions, we consider the stationary problem, with $f$ satisfying $(\mathrm{H})$ :

$$
\left(\mathrm{S}_{1}\right)\left\{\begin{array}{c}
(u q)^{\prime}-u^{\prime \prime}=0 \\
q^{\prime}+f(x, u)=0, \\
\text { with boundary conditions } \\
u(0)=\tilde{u}, \quad u^{\prime}(d)=q(d)=0,
\end{array}\right.
$$


with $\tilde{u} \in[0,1]$ and where primes denote differentation with respect to $x$. Clearly, $\tilde{u}=1$ and $\tilde{u}=0$ imply the trivial solutions $u=1, q=0$ and $u=0, q(x)=\int_{x}^{d} f(s, 0) \mathrm{d} s$, respectively. The latter corresponds to the physical situation when no salt is present in the system and the mangroves extract the maximal amount of water. We therefore restrict our discussion to the case $\tilde{u} \in(0,1)$. Integrating the first equation in Problem $\mathrm{S}_{1}$ over $(x, d)$, and using the boundary conditions, we obtain

$$
u^{\prime}(x)=u(x) q(x) \quad \text { for } x \in \Omega .
$$

Clearly $u \geqslant \tilde{u}>0$ in $\bar{\Omega}$. Therefore we can set

$$
w(x):=\log u(x) \quad \text { for } x \in \bar{\Omega},
$$

for which we find the boundary value problem

$$
\left(\mathrm{S}_{2}\right)\left\{\begin{array}{lr}
w^{\prime \prime}+g(x, w)=0 & \text { for } x \in \Omega, \\
w(0)=\log \tilde{u}, & w^{\prime}(d)=0,
\end{array}\right.
$$

with $g(x, w):=f\left(x, \mathrm{e}^{w}\right)$. As a consequence of $(\mathrm{H})$ the function $g$ satisfies:

$$
\left\{\begin{array}{l}
\text { (a) } g(x, \cdot) \in C((-\infty, 0]) \quad \text { for a.e. } x \in \Omega ; \\
\text { (b) } g(\cdot, s) \in L^{\infty}(\Omega) \quad \text { for all } s \in(-\infty, 0] ; \\
\text { (c) } g(x, \cdot) \text { is non-increasing in }(-\infty, 0] \text { and } g(x, 0)=0 \quad \text { for a.e. } x \in \Omega .
\end{array}\right.
$$

We can apply well known results (see, e.g. [5]) to prove the existence of solutions of Problem $\mathrm{S}_{2}$ in the class $W^{1,1}(\Omega)$. By the additional regularity in (b) it is straightforward to show that solutions of $\left(\mathrm{S}_{2}\right)$ belong to $W^{2, \infty}(\Omega)$ (note that $W^{1,1}(\Omega) \subset L^{\infty}(\Omega)$ in one space dimension). Finally, due to (c), we observe that the solution of Problem $\mathrm{S}_{2}$ depends monotonically in $\tilde{u}$. We also point out that the possible non-Lipschitz continuity of $f(x, \cdot)$ carries over to $g(x, \cdot)$. Consequences of this will be discussed in Section 6 which deals with the formation of dead cores.

When $f$ and $k$ are given by (2.13) and (2.14) or (2.15), qualitative statements about the behaviour of solutions can be made relatively easily. The reason is that in these cases the non-trivial part of a solution of Problem $\mathrm{S}_{2}$ is determined by two first-order autonomous equations (directly, when $k$ is as in (2.14), or after a straightforward transformation when $k$ is given by (2.15)). We briefly sketch the corresponding phase plane analysis.

Let $k$ be given by (2.14) with $x_{0}=1$ and $d>1$. In the interval $1 \leqslant x \leqslant d$, where no uptake of water takes place, we deduce directly

$$
q(x)=0 \quad \text { and } \quad u(x)=u(1) \quad \text { for } \quad x \in[1, d] .
$$

In the interval $(0,1)$ we consider the system

$$
\left\{\begin{array}{l}
w^{\prime}=q, \\
q^{\prime}=k_{0}\left(1-\mathrm{e}^{w}\right)^{p} .
\end{array}\right.
$$

Without giving the details of the phase plane analysis, we show in Fig. 1a (with $\tilde{u}=0.25, k_{0}=10$ and $p=1$ ) the result of the shooting procedure starting from $u(0)=\tilde{u}$ with $q(0)$ as shooting parameter. The behaviour of the orbits is typical for all values of $p \geqslant 1$. The right-hand side of 

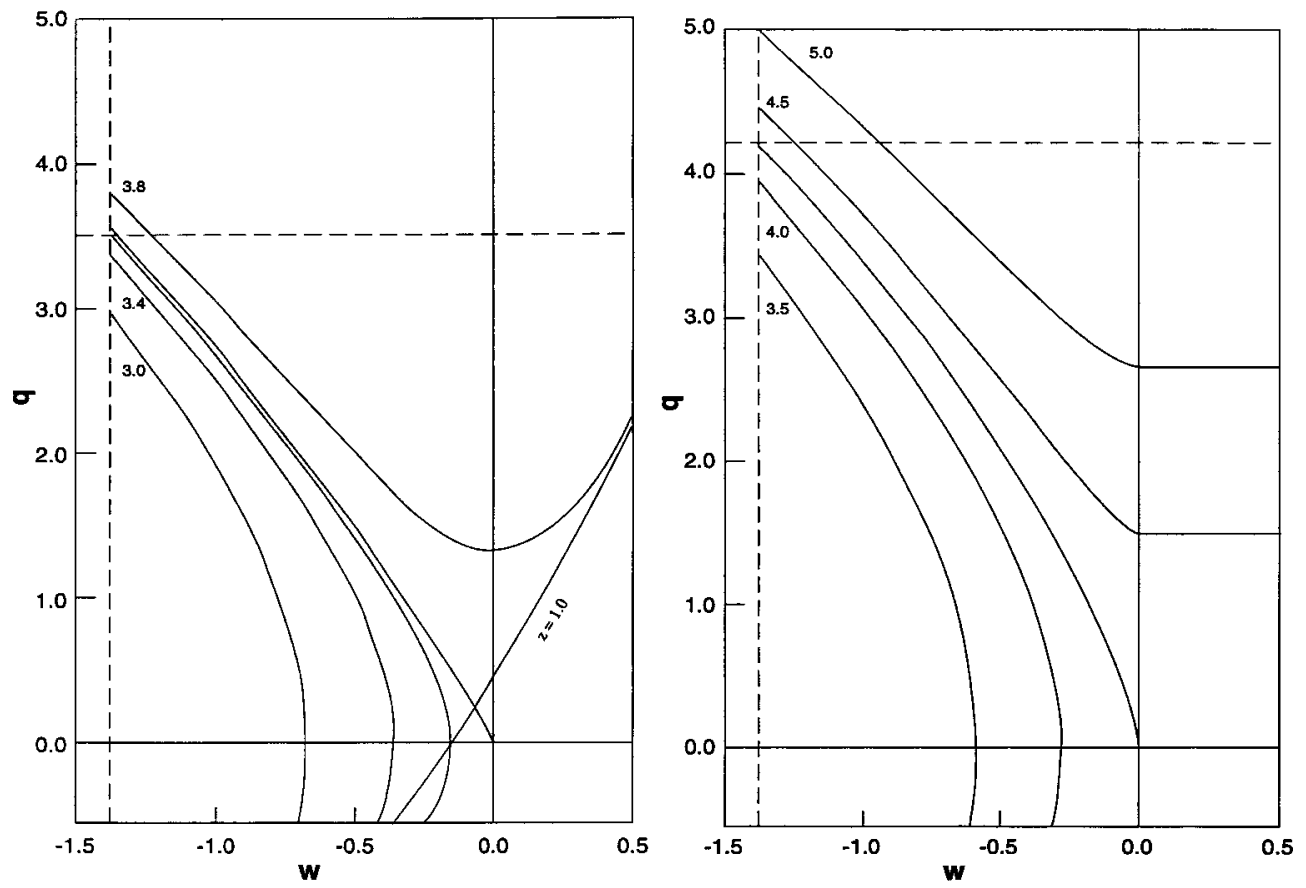

FIG. 1. Construction of the orbits. Parameters are $u_{0}=0.25, k_{0}=10$, and the root distribution $k(x)$ is given by (2.14). Numbers on curves are values of $q(0)$ used for shooting. The dashed lines indicate the values of $w(0)$ and $q(0)$ that are found. In the case $p=1$, the curve $\{z=1\}$ has been obtained numerically by shooting. (a) $p=1$. (b) $p=0.5$.

the second equation of (3.4) is smooth for that range of $p$, which means that the critical point $(w, q)=(0,0)$ (corresponding to $u=1, q=0)$ can never be reached at finite distance see, for instance, $[1,8]$. Consequently, the threshold concentration $u=1$ can never be attained. The desired orbit is the one that satisfies $q(1)=0$. The solution for $x>1$ is constant, see (3.3).

For $0<p<1$ the qualitative behaviour of the solution is drastically different. Then, the righthand side of the second equation of (3.4) loses its smoothness near $w=0$, implying that now the singular point $(0,0)$, or the threshold concentration, can be attained at finite depth. Note that the situation in the $w, q$ plane is quite different from the $p \geqslant 1$ case. This is shown in Fig. 1b, where $p=0.5$. For an appropriate choice of the parameters, the orbit will enter the origin at a distance less than or equal to $x=1$. That is to say, the threshold concentration $u=1$ occurs in or just below the mangrove root zone.

For other choices of the parameters a situation as in Fig. 1a may occur. Then the $q=0$ axis is reached at $x=1$ with $w(1)<0$, leading to a salt distribution that again can never attain the threshold value.

Next we consider $k$ given by (2.15). A direct phase plane analysis for system (3.4), which is now non-autonomous, or otherwise a reduction of Problem $\mathrm{S}_{1}$ to first integrals, seems not so transparent. However, because of the special form of $k$ we can transform (3.4) into an autonomous system which 
we can analyse as before. Set $v(x):=q(x)(1+x)$. Rewriting (3.4) in terms of $w$ and $v$ yields

$$
\left\{\begin{array}{l}
(1+x) w^{\prime}=v, \\
(1+x) v^{\prime}=v-k_{0}\left(1-\mathrm{e}^{w}\right)^{p} .
\end{array}\right.
$$

Changing the independent variable into

$$
s:=\log (1+x), \quad \text { when } \quad 0 \leqslant s \leqslant \log (1+d),
$$

we obtain

$$
\left\{\begin{array}{l}
\frac{\mathrm{d} w}{\mathrm{~d} s}=v, \\
\frac{\mathrm{d} v}{\mathrm{~d} s}=v-k_{0}\left(1-\mathrm{e}^{w}\right)^{p},
\end{array}\right.
$$

with $w(0)=\log \tilde{u}<0$ and $v(0)=u^{\prime}(0) / \tilde{u}$. Again the shooting procedure is: to find a value of $v(0)$, or equivalently of $u^{\prime}(0)$, so that the corresponding orbit intersects the $v=0$ axis, precisely when $s=\log (1+d)$. This implies $u^{\prime}(d)=0$, the desired boundary condition at $x=d$. A similar smoothness argument as mentioned previously gives that for all $p \geqslant 1$ an orbit cannot enter the origin $w=0, v=0$ at finite distance. Hence for all $p \geqslant 1$ we have $w(\log (1+d))<0$, implying that $u(d)<1$. Since $u$ is monotone in $x$, which follows from the positivity of $v$ along the appropriate orbit, we conclude that

$$
u(x)<u(d)<1 \quad \text { for } 0 \leqslant x \leqslant d \text { and for all } \quad p \geqslant 1 .
$$

Again this changes for $0<p<1$. As before, for certain parameter combinations we may find an orbit that reaches the origin at a distance $s^{*}<\log (1+d)$. This implies that

$$
u(x)=1 \quad \text { and } \quad q(x)=0 \quad \text { for } \quad x^{*}=\mathrm{e}^{s^{*}} \quad-1 \leqslant x \leqslant d
$$

For other parameter combinations the desired orbits intersects the $v=0$ axis as $s=\log (1+d)$ for negative $w$, giving for $u$ the inequalities from (3.7).

REMARK 3.1 In the case of an arbitrary root distribution function $k(x) \geqslant 0$, a simple reduction to a phase plane analysis as in the previous examples is not possible. Nevertheless, it is possible to analyse solutions of (3.4) qualitatively. The results are:

(1) If $p \geqslant 1$, then $u(x)<1$ for all $x \in[0, d]$. Hence no maximal concentration can occur in or below the root zone.

(2) If $p<1$, then $u=1$ in $\left[x_{0}, d\right]$ for some $x_{0} \in[0, d]$, is possible. As in the two examples, this depends on the value of the parameters $\tilde{u}, k_{0}$ and $d$.

(3) When comparing solutions corresponding to different root distribution functions, we have the following ordering: if $u_{i}(x)$ corresponds to the root distribution $k_{i}(x)$, for $i=1,2$, and if $k_{1}(x) \geqslant k_{2}(x)$ then $u_{1}(x) \geqslant u_{2}(x)$ for all $x \in[0, d]$.

A more detailed analysis concerning conditions under which the threshold concentration is reached is presented in Section 6. 


\section{The evolution problem}

In this section we study the mathematical setting of Problem P. In the time-dependent case, integration and change of variable as performed in Section 3 is no longer useful and a direct treatment of the coupled system is required. One of the main difficulties in studying Problem $\mathrm{P}$ is that, in general, the comparison principle does not hold. As a consequence, techniques involving comparison with sub- and super-solutions are not available and a more general approach must be considered. Using a fixed-point argument, we prove existence of a strong solution. Sufficient conditions for the uniqueness of solutions follow from a duality technique. We also give a counterexample for the comparison principle.

\subsection{Existence of solutions}

Because only boundedness of $f$ with respect to $x$ is required (see also example (2.14)), one cannot expect in general to find classical solutions. Therefore, we introduce the following class of strong solutions.

Definition 4.1 A pair $u: Q_{T} \rightarrow[0,1], q: Q_{T} \rightarrow \mathbb{R}$ is said to be a strong solution of Problem $P$ if

(i) for any $r<\infty$ and for $\mathcal{V}:=\left\{v \in W^{2, r}(\Omega): v(0)=0\right\}$,

$$
\begin{aligned}
& u \in u_{D}+W^{1, r}\left(0, T ; L^{r}(\Omega)\right) \cap L^{r}(0, T ; \mathcal{V}) \\
& q \in L^{\infty}\left(0, T ; W^{1, \infty}(\Omega)\right) .
\end{aligned}
$$

(ii) The differential equations and boundary conditions are satisfied almost everywhere, and (iii)

$$
\lim _{t \downarrow 0}\left\|u(x, t)-u_{0}(x)\right\|_{L^{2}(\Omega)}=0 .
$$

Concerning the data of Problem P we assume $u_{0}: \bar{\Omega} \rightarrow(0,1]$ and $u_{D}:[0, T] \rightarrow(0,1]$ such that

$$
u_{0} \in H^{1}(\Omega), \quad u_{D} \in H^{1}(0, T)
$$

and

$$
u_{D}(0)=u_{0}(0)
$$

Theorem 4.1 Let (H) and (4.2), (4.3) be satisfied. Then there exists a strong solution $(u, q)$ of Problem $\mathrm{P}$, for which

$$
\min \left\{\inf _{(0, T)} u_{D}, \inf _{\Omega} u_{0}\right\} \leqslant u \leqslant 1 \quad \text { a.e. in } Q_{T} .
$$

If $f(x, u)=k(x)(1-u)_{+}^{p}$ for some $p \in(0,1)$, and if $k, u_{0}, u_{D}$ are smooth enough, then

$$
\begin{aligned}
& u \in C^{2+p, 1+\frac{p}{2}}\left(\bar{Q}_{T}\right), \\
& q \in C^{1+p, p}\left(\bar{Q}_{T}\right) .
\end{aligned}
$$


Proof. The proof uses a fixed point argument. First we extend the domain of $f(x, \cdot)$ to $\mathbb{R}$ by setting for each $x \in \bar{\Omega}$,

$$
\tilde{f}(x, s):= \begin{cases}0 & \text { if } s>1, \\ f(x, s) & \text { if } 0 \leqslant s \leqslant 1, \\ f(x, 0) & \text { if } s<0,\end{cases}
$$

and we introduce Problem $\tilde{\mathrm{P}}$ by putting $f=\tilde{f}$ in Problem P. Fix $\alpha \in(2, \infty)$ and consider the set

$$
K:=\left\{(F, G) \in L^{2}\left(Q_{T^{*}}\right) \times L^{2}\left(Q_{T^{*}}\right):\|F\|_{L^{2}\left(Q_{T^{*}}\right)}<R,\|G\|_{L^{\alpha}\left(Q_{T^{*}}\right)}<\rho\right\},
$$

for certain positive numbers $T^{*}, R$ and $\rho$ which will be chosen later. Clearly, $K$ is a convex weakly compact subset of $L^{2}\left(Q_{T^{*}}\right) \times L^{2}\left(Q_{T^{*}}\right)$. We define the mapping $Q: K \rightarrow L^{2}\left(Q_{T^{*}}\right) \times L^{2}\left(Q_{T^{*}}\right)$ by

$$
Q(F, G):=\left(-(u q)_{x},-\tilde{f}(x, u)\right),
$$

where $u, q$ are solutions of

$$
\begin{cases}u_{t}-u_{x x}=F & \text { in } Q_{T^{*}}, \\ u(0, t)=u_{D}(t) & \text { in }\left(0, T^{*}\right) \\ u_{x}(d, t)=0 & \text { in }\left(0, T^{*}\right), \\ u(x, 0)=u_{0}(x) & \text { in } \Omega\end{cases}
$$

and

$$
\begin{cases}q_{x}=G & \text { in } Q_{T^{*}}, \\ q(d, t)=0 & \text { in }\left(0, T^{*}\right) .\end{cases}
$$

Note that a fixed point of $Q$ is a (local in time) solution of ( $(\tilde{\mathrm{P}})$. The regularity for $F, G, u_{0}$ and $u_{D}$ implies, see for instance [10], that (4.5) and (4.6) have unique solutions in the classes

$$
\begin{aligned}
& u \in H^{1}\left(0, T^{*} ; L^{2}(\Omega)\right) \cap L^{2}\left(0, T^{*} ; H^{2}(\Omega)\right) \cap L^{\infty}\left(0, T^{*} ; H^{1}(\Omega)\right), \\
& q \in L^{\alpha}\left(0, T^{*} ; W^{1, \alpha}(\Omega)\right) .
\end{aligned}
$$

In fact, replacing $F, G, u_{0}$ and $u_{D}$ by smooth approximations, testing the differential equation in (4.5) with $\left(u-u_{D}\right)_{t}$ and taking the limit yields the estimate

$$
\left\|u_{t}\right\|_{L^{2}\left(Q_{\tau}\right)}+\left\|u_{x}(\tau)\right\|_{L^{2}(\Omega)} \leqslant C\left\{\left\|u_{0}^{\prime}\right\|_{L^{2}(\Omega)}+\left\|u_{D}^{\prime}\right\|_{L^{2}\left(0, T^{*}\right)}+\|F\|_{L^{2}\left(Q_{\left.T^{*}\right)}\right.}\right\}
$$

which implies

$$
\|u(\tau)\|_{L^{\infty}(\Omega)} \leqslant u_{D}(\tau)+C\left\{\left\|u_{0}^{\prime}\right\|_{L^{2}(\Omega)}+\left\|u_{D}^{\prime}\right\|_{L^{2}\left(0, T^{*}\right)}+\|F\|_{L^{2}\left(Q_{T^{*}}\right)}\right\}
$$

for all $\tau \in\left(0, T^{*}\right)$. Note that using (4.8) we deduce from the differential equation in (4.5)

$$
\left\|u_{x x}\right\|_{L^{2}\left(Q_{\tau}\right)} \leqslant C\left\{\left\|u_{0}^{\prime}\right\|_{L^{2}(\Omega)}+\left\|u_{D}^{\prime}\right\|_{L^{2}\left(0, T^{*}\right)}+\|F\|_{L^{2}\left(Q_{T^{*}}\right)}\right\} .
$$

Clearly Problem (4.6) leads to

$$
\|q\|_{L^{\alpha}\left(0, T^{*} ; L^{\infty}(\Omega)\right)} \leqslant C\|G\|_{L^{\alpha}\left(Q_{T^{*}}\right)} .
$$

Finally, the regularity of $u$ implies that the initial data is satisfied in the sense of (4.1).

Having established the bounds of (4.5) and (4.6), we are now in a position to apply the fixed point theorem of [3]. For this we need to show: 
(i) $Q(K) \subset K$;

(ii) $Q$ is weakly-weakly sequentially continuous in $L^{2}\left(Q_{T^{*}}\right) \times L^{2}\left(Q_{T^{*}}\right)$.

To verify (i) we need to show that for appropiately chosen $T^{*}, R$, and $\rho$,

$$
\left\|(u q)_{x}\right\|_{L^{2}\left(Q_{T^{*}}\right)}<R \text { and }\|f(x, u)\|_{L^{\alpha}\left(Q_{T^{*}}\right)<\rho .}
$$

Using (4.8)-(4.11) we get

$$
\begin{aligned}
&\left\|(u q)_{x}\right\|_{L^{2}\left(Q_{T^{*}}\right.} \leqslant\left\|u_{x}\right\|_{L^{\infty}\left(0, T^{*} ; L^{2}(\Omega)\right)}\|q\|_{L^{2}\left(0, T^{*} ; L^{\infty}(\Omega)\right)}+\|u\|_{L^{\infty}\left(Q_{\left.T^{*}\right)}\right.}\left\|q_{x}\right\|_{L^{2}\left(Q_{\left.T^{*}\right)}\right.} \\
& \leqslant C\left\{\left\|u_{0}^{\prime}\right\|_{L^{2}(\Omega)}+\left\|u_{D}^{\prime}\right\|_{L^{2}\left(0, T^{*}\right)}+\|F\|_{L^{2}\left(Q_{\left.T^{*}\right)}\right.}\right\}\|G\|_{L^{\alpha}\left(Q_{\left.T^{*}\right)}\right.} \\
&<C\left\{\left\|u_{0}^{\prime}\right\|_{L^{2}(\Omega)}+\left\|u_{D}^{\prime}\right\|_{L^{2}\left(0, T^{*}\right)}+R\right\} \rho
\end{aligned}
$$

and from $(\mathrm{H})$ we obtain

$$
\|f(\cdot, u)\|_{L^{\alpha}\left(Q_{T^{*}}\right)} \leqslant c\left(T^{*}\right)^{1 / \alpha}\|f\|_{L^{\infty}(\Omega \times(0,1))} .
$$

We now fix $0<\rho<1 / C$ and $0<T^{*}<\rho^{\alpha}\left(c\|f\|_{L^{\infty}(\Omega \times(0,1))}\right)^{-\alpha}$. By choosing $R$ large enough, we can ensure that condition (4.12) is satisfied.

To verify (ii) one has to show that for every sequence $\left(F_{n}, G_{n}\right) \in K$ such that $\left(F_{n}, G_{n}\right) \rightarrow$ $(F, G) \in K$ weakly-weakly in $L^{2}\left(Q_{T^{*}}\right) \times L^{2}\left(Q_{T^{*}}\right)$, the images $Q\left(F_{n}, G_{n}\right)$ converge to $Q(F, G)$ also weakly-weakly in $L^{2}\left(Q_{T^{*}}\right) \times L^{2}\left(Q_{T^{*}}\right)$ (at least along a subsequence). Since $\left(F_{n}, G_{n}\right)$ is bounded in $L^{2}\left(Q_{T^{*}}\right) \times L^{\alpha}\left(Q_{T^{*}}\right)$ it follows that the solutions $\left(u_{n}, q_{n}\right)$ of (4.5) and (4.6) associated with these data satisfy uniform bounds of the type (4.8)-(4.11). Therefore, there exist subsequences, again denoted by the subindex $n$, and functions $u, q$, such that

$$
\begin{array}{ll}
u_{n} \rightarrow u & \text { weakly star in } L^{\infty}\left(Q_{\left.T^{*}\right),}\right. \\
u_{n} \rightarrow u & \text { weakly-star-weakly in } L^{\infty}\left(0, T ; H_{c}^{1}(\Omega)\right),
\end{array}
$$

with $H_{c}^{1}(\Omega):=\left\{v \in H^{1}(\Omega): v(0)=0\right\}$,

$$
u_{n t} \rightarrow u_{t}, \quad u_{n x x} \rightarrow u_{x x}, \quad \text { weakly in } L^{2}\left(Q_{T^{*}}\right)
$$

and

$$
q_{n x} \rightarrow q_{x} \quad \text { weakly in } \quad L^{\alpha}\left(Q_{T^{*}}\right)
$$

Applying [14] we deduce from (4.13), (4.14) that $u_{n} \rightarrow u$ strongly in $C\left([0, T] ; L^{2}(\Omega)\right)$ and strongly in $L^{2}\left(0, T ; H_{c}^{1}(\Omega)\right)$. Therefore, it also converges a.e. in $Q_{T}$, at least for a subsequence. Consequently, using the continuity of $\tilde{f}$,

$$
\tilde{f}\left(\cdot, u_{n}\right) \rightarrow \tilde{f}(\cdot, u) \quad \text { strongly in } L^{2}\left(Q_{T^{*}}\right) .
$$

Let $\varphi \in L^{2}\left(Q_{T^{*}}\right)$. Then

$$
\begin{aligned}
\int_{Q_{T^{*}}}\left(u_{n} q_{n}\right)_{x} \varphi & =\int_{Q_{T^{*}}} u_{n}\left(q_{n x}-q_{x}\right) \varphi+\int_{Q_{T^{*}}} u_{n} q_{x} \varphi+\int_{Q_{T^{*}}} u_{n x} q_{n} \varphi \\
& =: I_{1 n}+I_{2 n}+I_{3 n} .
\end{aligned}
$$


Using (4.15) and the observation that $u_{n} \rightarrow u$ strongly in $L^{r}\left(Q_{T^{*}}\right)$ for any $r \in[1, \infty)$ we find along a subsequence $n \rightarrow \infty$

$$
I_{1 n} \rightarrow 0 \quad \text { and } \quad I_{2 n} \rightarrow \int_{Q_{T^{*}}} u q_{x} \varphi .
$$

As a consequence of estimates (4.8) and (4.10), $u_{n x} \rightarrow u_{x}$ strongly in $L^{2}\left(Q_{T^{*}}\right)$ (see [12] ). Using further that $\left\|u_{x}\right\|_{L^{\infty}\left(0, T^{*} ; L^{2}(\Omega)\right)} \leqslant C$, we deduce $u_{n x} \rightarrow u_{x}$ strongly in $L^{r}\left(0, T^{*} ; L^{2}(\Omega)\right)$. Again with (4.15) this gives

$$
I_{3 n} \rightarrow \int_{Q_{T^{*}}} u_{x} q \varphi
$$

To conclude the proof of (ii) we note, by the linearity of Problems (4.5) and (4.6), that $u$ and $q$ are solutions corresponding to $F$ and $G$, respectively.

Hence, there exists a fixed point $(u, q)$ of $Q$ which is a local (in time) solution of $(\tilde{\mathrm{P}})$. By iterating the construction we can extend the solution to one which is global in time. Next we show that this solution satisfies Definition 4.1. Since $\tilde{f} \in L^{\infty}(\Omega \times \mathbb{R})$ the second equation of $(\tilde{\mathrm{P}})$ implies $q \in L^{\infty}\left(0, T ; W^{1, \infty}(\Omega)\right)$, and then, applying a bootstrap argument in $u$, the first equation yields $u_{t}-u_{x x} \in L^{\infty}\left(Q_{T}\right)$. Then the regularity stated in Definition 4.1 follows from well known results in [10].

Using $\varphi:=\min \{0, u\}$ and $\varphi:=\max \{0, u-1\}$ as test functions one easily shows that $u \geqslant 0$ and $u \leqslant 1$ in $\bar{Q}_{T}$. We note at this point that $0 \leqslant u \leqslant 1$ in $\bar{Q}_{T}$ implies $\tilde{f}(\cdot, u)=f(\cdot, u)$ in $\bar{Q}_{T}$ and therefore the pair $u, q$ is also a solution of Problem P. To show $\min \left\{\inf _{(0, T)} u_{D}, \inf _{\Omega} u_{0}\right\} \leqslant u$ we use $\tilde{f} \geqslant 0$ and the maximum principle. Indeed,

$$
u_{t}+q u_{x}-u_{x x} \geqslant u_{t}+q u_{x}-u_{x x}-u \tilde{f}(x, u)=0,
$$

and the maximum principle implies the assertion. Finally, the additional regularity of Theorem 4.1 follows from classical regularity theory for linear problems: for instance, see again [10].

\subsection{Uniqueness and comparison of solutions}

In many parabolic scalar problems involving monotone or Lipschitz-continuous nonlinearities, the uniqueness of solutions arises as a particular case of the comparison property. However, this situation, in general, changes for systems of equations, where the comparison principle may be violated even though uniqueness may still hold. In this section we show that this is the case for Problem P. We start with the following example.

Counter-example to the comparison property. Let $\phi:[0,1] \rightarrow[0,1]$ be a smooth function satisfying $\phi(0)=\phi(1)=\alpha \in(0,1)$, and $\phi^{\prime}(0)=\phi^{\prime \prime}(0)=\phi^{\prime}(1)=\phi^{\prime \prime}(1)=0$. Set $d=3$ and define the initial data $u_{10}, u_{20}:[0,3] \rightarrow[0,1]$ by

$$
u_{10}(x):=\left\{\begin{array}{ll}
\phi(x) & x \in[0,1], \\
0 & x \in(1,3],
\end{array} \quad u_{20}(x):= \begin{cases}\phi(x) & x \in[0,1], \\
0 & x \in(1,2), \\
\phi(3-x) & x \in[2,3] .\end{cases}\right.
$$

Note that $u_{10}, u_{20} \in C^{2}([0,3])$ and $u_{10} \leqslant u_{20}$ in $[0,3]$. Further, let $f(x, u)=1-u$ and $u_{D}(t) \equiv \alpha$, both $C^{\infty}$ functions in their domains. By Theorem 4.1 there exist solutions of $(\mathrm{P}),\left(u_{1}, q_{1}\right)$ and 
$\left(u_{2}, q_{2}\right)$, corresponding to these data such that $u_{1}, u_{2} \in C^{2,1}\left(\bar{Q}_{T}\right)$. At $t=0$ we have

$$
q_{1}(x, 0)-q_{2}(x, 0)=-\int_{x}^{3}\left(u_{10}(s)-u_{20}(s)\right) \mathrm{d} s .
$$

For $x \leqslant 2$ this expression is equal to the constant $c=\int_{0}^{1} \phi(x) \mathrm{d} x>0$. By subtracting the equations corresponding to $u_{1}$ and $u_{2}$ we find

$$
\left(u_{1}-u_{2}\right)_{t}=\left(u_{1}-u_{2}\right)_{x x}-\left(\left(u_{1}-u_{2}\right) q_{1}\right)_{x}-\left(u_{2}\left(q_{1}-q_{2}\right)\right)_{x},
$$

and if we consider this equation for $0<x<2$ and at $t=0$ we get

$$
\left(u_{1}-u_{2}\right)_{t}=-c u_{20}^{\prime} \text {. }
$$

Then it follows from the form of $u_{20}$ that $\left(u_{1}-u_{2}\right)_{t}$ cannot be non-positive everywhere on [0,2] at $t=0$, and that therefore we can find points $(x, t)$ such that $u_{1}(x, t)>u_{2}(x, t)$. This contradicts the comparison principle.

We show below, however, that uniqueness holds for this example. In fact, we demonstrate uniquenes for $f(x, \cdot)$ being Lipschitz continuous in $[0,1]$. For general, i.e. non-Lipschitz, functions $f(x, \cdot)$ we only have a partial result. It involves an additional condition on the component $u$. In Section 4.3 we show that the class of solutions satisfying this condition is non-empty.

\section{THEOREM 4.2}

(1) Uniqueness of solutions. Let $\left(u_{1}, q_{1}\right)$ and $\left(u_{2}, q_{2}\right)$ be two strong solutions of Problem $P$ and let $(H)$ be satisfied. If either

$$
f(x, \cdot) \quad \text { is Lipschitz continuous in }[0,1] \quad \text { for almost all } x \in \Omega,
$$

or any one of the solutions satisfies

$$
u(x, t)>\int_{0}^{x}\left|u_{x}(y, t)\right| \mathrm{d} y \quad \text { a.e. in } Q_{T},
$$

then $\left(u_{1}, q_{1}\right)=\left(u_{2}, q_{2}\right)$ a.e. in $Q_{T}$.

(2) Comparison of solutions. Assume now that $\left(u_{1}, q_{1}\right)$ and $\left(u_{2}, q_{2}\right)$ correspond to ordered data, i.e. $u_{1 D} \leqslant u_{2 D}$ in $(0, T)$ and $\left.u_{10}\right) \leqslant u_{20}$. Then, if

$$
u_{i x} \geqslant 0 \quad \text { in } Q_{T} \quad \text { and } \quad u_{i D}>0 \quad \text { in }(0, T],
$$

for either $i=1$ or $i=2$, then

$$
u_{1} \leqslant u_{2} \quad \text { and } \quad q_{1} \geqslant q_{2} \text { in } Q_{T} .
$$

\section{REMARK 4.1}

1. By (3.1) we have that the solution of the stationary problem is monotonic in space. Theorem 4.2 implies then that if $\left(u_{1 D}, u_{10}\right) \leqslant\left(v_{D}, v\right) \leqslant\left(u_{2 D}, u_{20}\right)$, where $v$ is the stationary solution corresponding to the boundary data $v_{D}$, then

$$
u_{1} \leqslant v \leqslant u_{2} \quad \text { and } \quad q_{1} \geqslant r \geqslant q_{2} \quad \text { in } Q_{T} .
$$


2. For both (4.16) and (4.17) it is unclear why they should influence the uniqueness of solutions. We believe that both are in fact only technical restrictions, and that uniqueness should hold under weaker assumptions on $f$ and $u$.

Proof of Theorem 4.2. We first discuss the proof of part (1). Let $\left(u_{1}, q_{1}\right)$ and $\left(u_{2}, q_{2}\right)$ be solutions of Problem $\mathrm{P}$ and set $(u, q):=\left(u_{1}-u_{2}, q_{1}-q_{2}\right)$. Then $(u, q)$ satisfies

$$
\left\{\begin{array}{c}
u_{t}+\left(u q_{1}+u_{2} q\right)_{x}-u_{x x}=0 \\
q_{x}+f\left(x, u_{1}\right)-f\left(x, u_{2}\right)=0
\end{array}\right\} \quad \text { a.e. in } Q_{T},
$$

Multiplying the differential equations of (4.19) by smooth functions $\varphi, \psi$ satisfying

$$
\varphi(0, t)=\varphi_{x}(d, t)=\psi(0, t)=0 \quad \text { for any } t \in[0, T],
$$

integrating in $Q_{\tau}$, with $\tau \in(0, T)$, and adding the resulting integral identities we obtain

$$
\begin{aligned}
\int_{\Omega} u(\tau) \varphi(\tau) & =\int_{Q_{\tau}} u\left[\varphi_{t}+q_{1} \varphi_{x}+\varphi_{x x}\right]-\int_{Q_{\tau}} q\left[\psi_{x}+u_{2 x} \varphi\right] \\
& +\int_{Q_{\tau}}\left(f\left(x, u_{1}\right)-f\left(x, u_{2}\right)\right)\left[u_{2} \varphi+\psi\right] .
\end{aligned}
$$

We consider the function

$$
h(x, t):= \begin{cases}\frac{f\left(x, u_{1}\right)-f\left(x, u_{2}\right)}{u} & \text { if } u \neq 0 \\ 0 & \text { if } u=0\end{cases}
$$

which is non-positive because $f(x, \cdot)$ is non-increasing. For $m \in \mathbb{N}, m \geqslant 1$, we consider the functions $h H(h+m)$, where $H$ denotes the Heaviside function: $H(s)=1$ for $s \geqslant 0, H(s)=0$ for $s \leqslant 0$. We regularize these functions in such a way that we obtain a smooth sequence $\left\{h^{m}\right\} \subset$ $C^{2}\left(Q_{\tau}\right)$ satisfying

(i) $h^{m+1} \leqslant h^{m}$ in $Q_{\tau}$,

(ii) $0 \geqslant h^{m} \geqslant \max \{-m, h\}$,

(iii) $h^{m} \rightarrow h$ a.e. in $Q_{\tau}$.

The regularity of solutions of Problem $\mathrm{P}$ allows us to introduce sequences $\left\{q_{1}^{n}\right\}_{n} \geqslant 1,\left\{u_{2}^{n}\right\}_{n} \geqslant 1 \subset$ $C^{2}\left(Q_{T}\right)$ such that

$$
q_{1}^{n} \rightarrow q_{1} \quad \text { and } \quad u_{2}^{n} \rightarrow u_{2} \quad \text { strongly in } L^{2}\left(0, T ; H^{1}(\Omega)\right)
$$

as $n \rightarrow \infty$ with

$$
\begin{aligned}
& \varlimsup{ }_{\lim }\left\|q_{1}^{n}\right\|_{L^{\infty}\left(Q_{T}\right)} \leqslant\left\|q_{1}\right\|_{L^{\infty}\left(Q_{T}\right)}, \quad \overline{\lim }\left\|q_{1 x}^{n}\right\|_{L^{2}\left(Q_{T}\right)} \leqslant\left\|q_{1 x}\right\|_{L^{2}\left(Q_{T}\right)}, \\
& \varlimsup\left\|u_{2}^{n}\right\|_{L^{\infty}\left(Q_{T}\right)} \leqslant\left\|u_{2}\right\|_{L^{\infty}\left(Q_{T}\right)}, \quad \overline{\lim }\left\|u_{2 x}^{n}\right\|_{L^{2}\left(Q_{T}\right)} \leqslant\left\|u_{2 x}\right\|_{L^{2}\left(Q_{T}\right)}
\end{aligned}
$$


and $u_{2}^{n}$ satisfying (4.17). Using these approximations we rewrite (4.21) as

$$
\begin{aligned}
\int_{\Omega} u(\tau) \varphi(\tau) & =\int_{Q_{\tau}} u\left[\varphi_{t}+q_{1}^{n} \varphi_{x}+\varphi_{x x}+h^{m}\left(u_{2}^{n} \varphi+\psi\right)\right]-\int_{Q_{\tau}} q\left[\psi_{x}+u_{2 x}^{n} \varphi\right] \\
& +\int_{Q_{\tau}} u\left(h-h^{m}\right)\left(u_{2} \varphi+\psi\right)-\int_{Q_{\tau}} u_{x}\left(q_{1}-q_{1}^{n}\right) \varphi \\
& -\int_{Q_{\tau}} u\left(q_{1 x}-q_{1 x}^{n}\right) \varphi+\int_{Q_{\tau}} u h^{m}\left(u_{2}-u_{2}^{n}\right) \varphi-\int_{Q_{\tau}} q\left(u_{2 x}-u_{2 x}^{n}\right) \varphi .
\end{aligned}
$$

Next we select the functions $\varphi$ and $\psi$, being the solutions of

$$
\left\{\begin{array}{c}
\varphi_{t}+q_{1}^{n} \varphi_{x}+\varphi_{x x}+h^{m}\left(u_{2}^{n} \varphi+\psi\right)=0 \\
\psi_{x}+u_{2 x}^{n} \varphi=0 \\
\varphi(\tau)=\xi \text { in } \Omega
\end{array}\right\} \quad \text { in } Q_{\tau},
$$

with $\varphi, \psi$ satisfying (4.20) and with $\xi \in C_{0}^{\infty}(\Omega), \xi \geqslant 0$.

\section{LEMMA 4.3}

(i) Assume either (4.16) or (4.17). Then, for each $n$ and $m$ there exists a unique solution $\varphi, \psi \in$ $C^{2,1}\left(\bar{Q}_{\tau}\right)$ of (4.26) such that $\|\varphi\|_{L^{\infty}\left(Q_{\tau}\right)}$ and $\|\psi\|_{L^{\infty}\left(Q_{\tau}\right)}$ are uniformly bounded with respect to $n$ and $m$.

(ii) Assume (4.18). Then, in addition to the uniform bounds we have

$$
\varphi \geqslant 0 \quad \text { in } Q_{T} \quad \text { and } \quad \varphi_{x}(0, t) \geqslant 0 \quad \text { in }(0, T) .
$$

End of proof of Theorem 4.2. Using the functions provided by Lemma 4.3 we obtain from (4.25)

$$
\begin{aligned}
\int_{\Omega} u(\tau) \xi=\int_{Q_{\tau}} u\left(h-h^{m}\right)\left(u_{2} \varphi+\psi\right)-\int_{Q_{\tau}} u_{x}\left(q_{1}-q_{1}^{n}\right) \varphi \\
\quad-\int_{Q_{\tau}} u\left(q_{1 x}-q_{1 x}^{n}\right) \varphi+\int_{Q_{\tau}} u h^{m}\left(u_{2}-u_{2}^{n}\right) \varphi-\int_{Q_{\tau}} q\left(u_{2 x}-u_{2 x}^{n}\right) \varphi .
\end{aligned}
$$

Finally, by the uniform estimates from Lemma 4.3 and (4.24), we can pass to the limit in (4.28) and obtain for $n \rightarrow \infty$

$$
\int_{\Omega} u(\tau) \xi=\int_{Q_{\tau}} u\left(h-h^{m}\right)\left(u_{2} \varphi+\psi\right) .
$$

Using Lemma 4.3 again and the convergence properties of the sequence $\left\{h^{m}\right\}$ we find

$$
\int_{Q_{\tau}} u\left(h-h^{m}\right)\left(u_{2} \varphi+\psi\right) \rightarrow 0 \quad \text { as } \quad m \rightarrow \infty,
$$

and hence we obtain from (4.29)

$$
\int_{\Omega} u(\tau) \xi=0
$$


for any test function $\xi \geqslant 0$. We therefore deduce that $u_{1}=u_{2}$ a.e. in $Q_{\tau}$ for any $\tau \in(0, T)$. Checking that this implies $q_{1}=q_{2}$ is straightforward.

To prove part (2) we define again $(u, q):=\left(u_{1}-u_{2}, q_{1}-q_{2}\right)$ and consider the problem for $(u, q)$ given by (4.19) but with $u_{D}:=u_{1 D}-u_{2 D} \leqslant 0$ and $u_{0}:=u_{10}-u_{20} \leqslant 0$. Following the proof of part (1) we get

$$
\int_{\Omega} u(\tau) \varphi(\tau)=\int_{\Omega} u_{0} \varphi(0)+\int_{0}^{T} u_{D}(t) \varphi_{x}(0, t) \mathrm{d} t+I_{1},
$$

with $I_{1}$ given by the right-hand side of (4.25). By Lemma 4.3 the solution $(\varphi, \psi)$ of (4.26) satisfies $\varphi(0, t) \geqslant 0$ and $\varphi_{x}(0, t) \geqslant 0$. Hence, we obtain from (4.32) and (4.26)

$$
\int_{\Omega} u(\tau) \xi \leqslant 0
$$

for all $\xi \geqslant 0$, from which the assertion follows.

Proof of Lemma 4.3. Because (4.26) is linear with smooth coefficients and data, the existence, uniqueness and regularity of solutions is well known [10]. To show the uniform $L^{\infty}$ bounds we consider separately the cases (4.16) and (4.17). If (4.16) holds, then $h$ defined by (4.22) is bounded, and consequently, $\left\{h^{m}\right\}$ is uniformly bounded in $L^{\infty}\left(Q_{\tau}\right)$ with respect to $m$. Further, (4.24) ensures that $q_{1}^{n}, u_{2}^{n}$ and $u_{2 x}^{n}$ are uniformly bounded in $L^{\infty}\left(Q_{\tau}\right)$ with respect to $n$.

Next assume (4.17). We assert that the global maximum of $|\varphi|$ is attained either at the boundary $x=0$ or initially at $t=\tau$ implying $\|\varphi\|_{L^{\infty}\left(Q_{\tau}\right)}$ uniformly bounded with respect to $m$ and $n$. Suppose this is not true. Let $\left(x_{0}, \tau_{0}\right) \in Q_{\tau}$ be the point where the global maximum of $|\varphi|$ is attained. Then $\left(x_{0}, \tau_{0}\right)$ is either a point of global maximum or a point of global minimum for $\varphi$. Let us consider first the case in which $\left(x_{0}, \tau_{0}\right)$ is a point of global maximum. The boundary data for $\varphi$ implies $\varphi\left(x_{0}, \tau_{0}\right)>0$ and the $\varphi$-equation in (4.26) yields, using $h^{m} \leqslant 0$,

$$
u_{2}^{n}\left(x_{0}, \tau_{0}\right) \varphi\left(x_{0}, \tau_{0}\right)+\psi\left(x_{0}, \tau_{0}\right) \leqslant 0 .
$$

Integrating the $\psi$-equation of (4.26) in $(0, x)$ gives

$$
\psi(x, t)=\int_{0}^{x}\left(-u_{2 x}^{n}(y, t)\right) \varphi(y, t) \mathrm{d} y .
$$

Therefore, from (4.33), (4.34) and assumption (4.17) we obtain

$$
\begin{aligned}
u_{2}^{n}\left(x_{0}, \tau_{0}\right) \varphi\left(x_{0}, \tau_{0}\right) & \leqslant \int_{0}^{x_{0}} u_{2 x}^{n}\left(y, \tau_{0}\right) \varphi\left(y, \tau_{0}\right) \mathrm{d} y \\
& \leqslant \int_{0}^{x_{0}}\left|u_{2 x}^{n}\left(y, \tau_{0}\right)\right| \mathrm{d} y \sup _{y \in\left(0, x_{0}\right)}\left|\varphi\left(y, \tau_{0}\right)\right| \\
& =\int_{0}^{x_{0}}\left|u_{2 x}^{n}\left(y, \tau_{0}\right)\right| \mathrm{d} y \varphi\left(x_{0}, \tau_{0}\right)<u_{2}^{n}\left(x_{0}, \tau_{0}\right) \varphi\left(x_{0}, \tau_{0}\right),
\end{aligned}
$$

a contradiction. If the global maximum is attained at a point $\left(d, \tau_{0}\right)$, then by the strong maximum principle $\varphi_{x}\left(d, \tau_{0}\right)>0$. This gives again a contradiction. Finally, if $\left(x_{0}, \tau_{0}\right)$ is a point of global 
minimum for $\varphi$, we may repeat the argument above, obtaining a similar contradiction. To finish the proof of (i) we use (4.34) and (4.24) to find

$$
\|\psi\|_{L^{\infty}\left(Q_{\tau}\right)} \leqslant\|\varphi\|_{L^{\infty}\left(Q_{\tau}\right)}\left\|u_{2}\right\|_{L^{\infty}\left(0, \tau ; W^{1,1}(\Omega)\right)},
$$

which is also independent of $m$ and $n$.

The proof of (ii) follows the same ideas as that of (i). We assume (4.18) and assert that the global minimum of $\varphi$ is attained either at the boundary $x=0$ or initially at $t=\tau$ implying (4.27), see (4.20) and (4.26). Suppose this is not true. Then, using the arguments of part (i) for the function $\varphi$ instead of $|\varphi|$, we are led to an expression similar to (4.35):

$$
\begin{aligned}
u_{2}^{n}\left(x_{0}, \tau_{0}\right) \varphi\left(x_{0}, \tau_{0}\right) & \geqslant \int_{0}^{x_{0}} u_{2 x}^{n}\left(y, \tau_{0}\right) \varphi\left(y, \tau_{0}\right) \mathrm{d} y \\
& \geqslant \int_{0}^{x_{0}} u_{2 x}^{n}\left(y, \tau_{0}\right) \mathrm{d} y \inf _{y \in\left(0, x_{0}\right)} \varphi\left(y, \tau_{0}\right)>u_{2}^{n}\left(x_{0}, \tau_{0}\right) \varphi\left(x_{0}, \tau_{0}\right),
\end{aligned}
$$

a contradition.

\subsection{Condition (4.17)}

Since inequality (4.17) is difficult to verify directly, the following observation is useful.

Proposition 4.4 Let $u_{\min }>0$ be given by (4.4). If there exists $L>0$ such that

$$
u_{x} \leqslant L \quad \text { in } Q_{T}, \quad \text { with } L<\frac{u_{\min }}{2 d}
$$

then (4.17) is satisfied.

Proof. Define $w(x, t):=u(x, t)-L x$ for $(x, t) \in Q_{T}$. Then $w_{x} \leqslant 0$ in $Q_{T}$ and $\left|u_{x}\right| \leqslant-w_{x}+L$, implying

$$
\int_{0}^{x}\left|u_{x}\right| \leqslant u_{D}-u+2 L d \quad \text { in } Q_{T} .
$$

Using (4.4) and (4.36) we obtain

$$
\int_{0}^{x}\left|u_{x}\right| \leqslant 2 L d<u_{\min } \leqslant u \quad \text { in } Q_{T} .
$$

Clearly, the condition of Proposition 4.4 is stronger than (4.17). However, the following example shows that it is relatively straightforward to obtain.

Proposition 4.5 Let $f(x, s):=k(x)(1-s)_{+}^{p}$, with $k \geqslant 0$ and $k^{\prime} \leqslant 0$ a.e. in $\Omega$. Assume $u_{0} \equiv u_{D}=\tilde{u}$, with

$$
\tilde{u}>\max \left\{\frac{2}{2+p}, 1-\left(d^{2} k(0)\right)^{-p}\right\} .
$$

Then (4.36) holds. 
Proof. We regularize $f$ by $f_{n}$, with $\left\{f_{n}\right\}$ a sequence of smooth functions converging to $f$ stronglyuniformly in $L^{r}(\Omega) \times C([0,1])$, for any $r<\infty$, and with the following properties:

$$
\left\{\begin{array}{l}
n^{-1} \leqslant f_{n} \leqslant f+n^{-1} \\
f_{n x} \leqslant 0
\end{array} \quad \text { in } \Omega \times[0,1] \quad \text { and } \quad 2 f_{n}+u_{n} f_{n u}<0 \quad \text { in } Q_{T} .\right.
$$

It is easy to see that due to the special form of $f$ assumed in the statement of the proposition such a sequence exists. We then consider the sequence of regularized problems $\left\{\mathrm{P}_{n}\right\}$ and their solutions $\left\{u_{n}, q_{n}\right\}$. Note that Theorem 4.1 implies $u_{n} \geqslant \tilde{u}$ in $Q_{T}$. Differentiating the first equation of Problem $\mathrm{P}_{n}$ with respect to $x$, we obtain the following problem for $v_{n}:=u_{n x}$ :

$$
v_{n t}+q_{n} v_{n x}-v_{n x x}-\left(2 f_{n}+u_{n} f_{n u}\right) v_{n}=f_{n x} u_{n} \quad \text { in } Q_{T},
$$

with

$$
\begin{cases}v_{n}(0, t) \geqslant 0 & \text { in }(0, T), \\ v_{n}(d, t)=0 & \text { in }(0, T), \\ v_{n}(x, 0)=0 & \text { in }(0, d) .\end{cases}
$$

Applying the maximum principle to (4.39) we deduce that the maximum of $v_{n}$ is at $x=0$. We now consider the solution $\left(U_{n}, Q_{n}\right)$ of the stationary problem $\left(\mathrm{S}_{n}\right)$ corresponding to $\left(\mathrm{P}_{n}\right)$ and use Theorem 4.2 to deduce $U_{n} \geqslant u_{n}$ in $Q_{T}$, and therefore $U_{n x}(0) \geqslant u_{n x}(0, t)$ in $(0, T)$. We then obtain, see (3.1),

$$
u_{n x}(x, t) \leqslant U_{n x}(0)=U_{n}(0) Q_{n}(0)
$$

and then, by (4.38),

$$
u_{n x}(x, t) \leqslant \tilde{u} \int_{0}^{d} f_{n}(y, U(y)) \mathrm{d} y \leqslant d \tilde{u}\left[(1-\tilde{u})^{p} k(0)+\frac{1}{n}\right] .
$$

To pass to the limit we note that the bounds obtained in Theorem 4.1 are valid for Problem $\mathrm{P}_{n}$, uniformly in $n$. Therefore the sequence $\left(u_{n}, q_{n}\right)$ converges pointwise to $(u, q)$. To show that this limit is a solution of Problem P only requires to consider the limit of $f_{n}\left(\cdot, u_{n}\right)$ since the other terms are treated as in Theorem 4.1. But this is a consequence of the pointwise convergence of $u_{n}$ to $u$ and the strong-uniform convergence of $f_{n}$ to $f$ in $L^{r}(\Omega) \times C([0,1])$. Note that (4.40) gives $u_{x}(x, t) \leqslant d \tilde{u}(1-\tilde{u})^{p} k(0)$, which implies (4.36).

\section{Qualitative properties}

In this section we derive some properties of solutions of Problem P. For simplicity we confine ourselves to the case of constant boundary data, i.e.

$$
u_{D} \equiv \tilde{u} \in(0,1),
$$

and let $(U, Q)$ be the stationary solution corresponding to this boundary condition. Since $U$ is increasing in $x$, it is admissible as a comparison function by Theorem 4.2: if the initial data, $u_{0}$, and $U$ are ordered, e.g. $u_{0} \leqslant U$, then this ordering persists through time: $u(x, t) \leqslant U(x)$ for all $x \in \Omega$ and $t>0$. This property allows us to prove the convergence for $t \rightarrow \infty$ to the stationary state. 
Theorem 5.1 Let $(u, q)$ be a solution of Problem $P$ and let $(U, Q)$ be the corresponding steady state solution of Problem $S_{1}$. Let $u_{0}$ and $U$ be ordered, i.e. either $u_{0}(x) \leqslant U(x)$ for all $x \in \Omega$, or $u_{0}(x) \geqslant U(x)$ for all $x \in \Omega$. Then

$$
\begin{aligned}
& u(\cdot, t) \rightarrow U \\
& q(\cdot, t) \rightarrow Q
\end{aligned} \quad \text { as } t \rightarrow \infty,
$$

uniformly in $\Omega$.

Proof. For the proof we assume that $u_{0} \leqslant U$; for the opposite inequality the argument is identical. Writing

$$
q(0, t)=\int_{\Omega} f(x, u(x, t)) \mathrm{d} x,
$$

the inequality $u(\cdot, t) \leqslant U$, provided by Theorem 4.2, implies that $q(0, t) \geqslant Q(0)$. It also follows from this inequality that $u_{x}(0, t) \leqslant U_{x}(0)$. By integrating the first equation of Problem $\mathrm{P}$ we find

$$
\begin{aligned}
\frac{\mathrm{d}}{\mathrm{d} t} \int_{\Omega} u(x, t) \mathrm{d} x & =-u_{x}(0, t)+\tilde{u} q(0, t) \\
& \geqslant-U_{x}(0)+\tilde{u} Q(0)=0 .
\end{aligned}
$$

The last equality follows from the equation satified by $(U, Q)$ and the boundary conditions $U_{x}(d)=$ $Q(d)=0$. This implies that the mass $\int u$ is a Lyapunov function, and even a strict Lyapunov function if $q(0, t)>Q(0)$.

We next derive a priori estimates that provide the necessary compactness. The function $w=$ $u-\tilde{u}$ satisfies the equation

$$
w_{t}+w_{x} q-(w+\tilde{u}) f(\cdot, w+\tilde{u})-w_{x x}=0,
$$

and by a standard partial integration we derive from this equation the energy inequality

$$
\begin{aligned}
\frac{1}{2} \int_{\Omega} w(x, \tau+1)^{2} \mathrm{~d} x+\int_{\tau}^{\tau+1} \int_{\Omega} w_{x}^{2} \leqslant \frac{1}{2} \int_{\Omega} w(x, \tau)^{2} \mathrm{~d} x & \\
& +\int_{\tau}^{\tau+1} \int_{\Omega}\left(w+\tilde{u}-\frac{1}{2} w^{2}\right) f(\cdot, w+\tilde{u}) .
\end{aligned}
$$

Since $w$ is bounded, it follows that there exists a constant $C$, independent of $\tau$, such that

$$
\int_{\tau}^{\tau+1} \int_{\Omega} w_{x}^{2} \leqslant C .
$$

Therefore there exists a sequence $\tau_{n} \rightarrow \infty$, with $\left|\tau_{n+1}-\tau_{n}\right| \leqslant 2$, such that

$$
\int_{\Omega} u_{x}^{2}\left(x, \tau_{n}\right) \mathrm{d} x \leqslant C,
$$

and by combining this sequence with the argument of the existence proof (Theorem 4.1) it follows that the bounds on $u$ and $q$ in the spaces $L^{\infty}\left(0, T ; H^{1}(\Omega)\right)$ and $L^{\infty}\left(0, T ; W^{1, \infty}(\Omega)\right)$ are independent of $T$. 
We choose a sequence $t_{n} \rightarrow \infty$ such that $(u, q)\left(\cdot, t_{n}\right)$ converges uniformly to a limit $\left(u_{\infty}, q_{\infty}\right)$. Standard Lyapunov arguments imply that the mass is constant for the solution with initial data $u_{\infty}$. The final part of this proof consists of showing that this property implies that $u_{\infty}=U$.

To do this, we now switch to the solution $(v, r)$ of Problem $\mathrm{P}$ which has $u_{\infty}$ as its initial datum. Note that by construction $u_{\infty} \leqslant U$ and therefore $v(\cdot, t) \leqslant U$ for all $t \geqslant 0$. As remarked above, the mass is a strictly increasing Lyapunov function if $r(0, t)>Q(0)$, in which case a contradiction is obtained and the proof is complete. We therefore only need to concentrate on the situation $v(\cdot, t) \not \equiv$ $U$, but $r(0, t)=Q(0)$ for all $t$. It follows from the latter equality and $v(\cdot, t) \leqslant U$ that $r(x, t)=$ $Q(x)$ for all $x$ and $t$; therefore $v$ and $U$ satisfy

$$
v_{t}+(v Q)_{x}-v_{x x}=0 \quad \text { and } \quad U_{t}+(U Q)_{x}-U_{x x}=0 .
$$

By hypothesis $v(\cdot, t) \leqslant U$ for all $t \geqslant 0$, and $v(\cdot, t) \not \equiv U$; it follows from the boundary point lemma that $v_{x}(0, t)<U_{x}(0)$ for all $t>0$. At the same time, the fact that the mass $\int v$ remains constant implies (by (5.2)) that $v_{x}(0, t)=\tilde{u} Q(0)$. This contradicts the properties of $U$ and $Q$ :

$$
0<U_{x}(0)-v_{x}(0, t)=\tilde{u} Q(0)-\tilde{u} Q(0)=0 .
$$

This concludes the proof of the Theorem.

In the remainder of this section we consider the special case when $f$ is given by

$$
f(x, u):=k(x)(1-u)_{+}^{p}, \quad 0<p<1,
$$

with $k$ satisfying (2.14). Of particular interest here is the formation of the dead core $\{u=1\}$, where the salt concentration reaches its maximum value and when the uptake of water by the roots of the mangroves stops. In Section 6 we will discuss the existence of dead cores for both the steady and time-dependent problem. In particular we show that a dead core can be formed in finite time. Here we show the opposite. By using appropiate super-solutions we will estimate the set $\{u<1\}$. To simplify the discussion we assume for the rest of this section that $u_{0}=\tilde{u}$.

Let $(U, Q)$ be the solution of Problem $\mathrm{S}_{1}$ and suppose parameters are chosen such that

$$
U(x)<1 \quad \text { if } x \in\left[0, x_{0}\right) \quad \text { and } \quad U(x)=1 \quad \text { if } x \in\left[x_{0}, d\right]
$$

with $x_{0} \leqslant 1$. As a first observation we have the following proposition.

Proposition $5.2 u<1$ in $(1, d] \times[0, T]$.

Proof. In the set $(1, d) \times(0, T)$ we have

$$
u_{t}-u_{x x}=0, \quad q=0
$$

and

$$
u(1, t) \in[\tilde{u}, 1], \quad u_{x}(d, t)=0, \quad u(x, 0)=\tilde{u} .
$$

We construct an explicit supersolution on $(1, d) \times(0, T]$ :

$$
\phi(x, t)=1-(1-\tilde{u}) \exp \left(-\frac{\pi^{2}}{4(d-1)^{2}} t\right) \sin \left(\frac{\pi}{2} \frac{x-1}{d-1}\right) .
$$

It follows that $u(x, t) \leqslant \phi(x, t)<1$ on $(1, d] \times[0, T]$. 
Using the steady-state solution we find the following proposition.

Proposition $5.3 u<1$ in $\left[0, x_{0}\right) \times[0, T]$.

Proof. Theorem 4.2 gives $u \leqslant U$ in $Q_{T}$. Using (5.4) the assertion follows.

Finally, we use a time-dependent super-solution to show the following proposition.

Proposition 5.4 There exists $T_{0}>0$ such that $u<1$ in $[0, d] \times\left[0, T_{0}\right]$.

Proof. Let $\xi:[0, \infty) \rightarrow \mathbb{R}$ be the solution of

$$
\left\{\begin{array}{l}
\dot{\xi}=k_{0} \xi(1-\xi)_{+}^{p} \quad t>0 \\
\xi(0)=\tilde{u} .
\end{array}\right.
$$

Clearly, there exists $T_{0}>0$ such that $\tilde{u}<\xi<1$ and $\dot{\xi}>0$ in $\left(0, T_{0}\right)$, and $\xi=1$ in $\left[T_{0}, \infty\right)$. The pair

$$
\begin{aligned}
& \bar{u}(x, t):=\xi(t), \\
& \bar{q}(x, t):=\int_{x}^{d} f(y, \xi(t)) \mathrm{d} y
\end{aligned}
$$

is a solution of Problem P with data $\bar{u}_{D}=\xi \geqslant \tilde{u}$ and $\bar{u}_{0}=\tilde{u}$. Since $\bar{u}$ is constant in space, condition (4.18) is trivially satisfied. Then, by Theorem 4.2 we have

$$
u(x, t) \leqslant \xi(t) \quad \text { for }(x, t) \in Q_{T},
$$

which proves the assertion of the proposition.

\section{Formation of a dead core}

In this section we present results concerning the formation of dead cores in the stationary and timedependent problems. For the stationary problem we use the comparison principle to give accurate conditions on the data implying the existence of a dead core. For the time-dependent problem the comparison principle can provide some results on the occurrence of dead cores (see the previous section), but to obtain more general results we need to apply more sophisticated techniques.

We therefore use a local energy method for free boundary problems [2,7]. First we introduce an energy functional given in terms of the norms of the natural energy spaces associated with the problem. Then we obtain a differential inequality for such a functional and, finally, we deduce the formation of a dead core from the properties of this inequality.

\subsection{The steady state problem}

To study conditions implying the formation of a dead core in the stationary problem we consider the formulation $\left(\mathbf{S}_{2}\right)$. Note that, by the maximum principle, the solution satisfies $\log \tilde{u} \leqslant w \leqslant 0$ in $\Omega$, and that the dead core, if it does exist, is given by the set $\{x \in[0, d]: w(x)=0\}$. We will define a subsolution of Problem $\mathrm{S}_{2}$ vanishing in a subset of $\Omega$ and use the comparison principle to deduce the same property for the solution of Problem $\mathrm{S}_{2}$. We assume, in addition to $(\mathrm{H})$, the following structural property on $f$ :

$$
f(\cdot, s) \geqslant k_{0}(1-s)^{p}, \quad \text { for } s \in[0,1],
$$

in $(0, d)$, with $k_{0}$ a positive constant and $p \in(0,1)$. 
THEOREM 6.1 Assume (6.1) and

$$
-\frac{\log \tilde{u}}{(1-\tilde{u})^{p}}<k_{0} d^{2} \frac{(1-p)^{2}}{2(1+p)} .
$$

Then $\{x: w(x)=0\} \supset\left(x_{0}, d\right)$, with $x_{0}$ given by (6.4).

Proof. We consider the following subsolution of Problem $\mathrm{S}_{2}$ :

$$
\underline{w}(x):= \begin{cases}a\left(x_{0}-x\right)^{\frac{2}{1-p}} & \text { if } x \in\left(0, x_{0}\right), \\ 0 & \text { if } x \in\left(x_{0}, d\right)\end{cases}
$$

with

$$
a:=-\left(k_{0}\left|\frac{1-\tilde{u}}{\log \tilde{u}}\right|^{p} \frac{(1-p)^{2}}{2(1+p)}\right)^{\frac{1}{1-p}} \text { and } \quad x_{0}:=\left(\frac{\log \tilde{u}}{a}\right)^{\frac{1-p}{2}} .
$$

It is easy to check that if (6.2) holds then $x_{0}<d$ from where the result follows by applying the maximum principle.

REMARK 6.1 The result of Theorem 6.1 may be localized. Assuming that (6.1) holds in an interval $\left(z_{0}, z_{1}\right) \subset(0, d)$ then we can define a local subsolution by (6.3) with $a$ given by (6.4) but $x_{0}$ redefined by $x_{0}:=z_{0}+\left(\frac{\log \tilde{u}}{a}\right)^{(1-p) / 2}$. These conditions imply $\underline{w} \leqslant w$ in $\left(z_{0}, z_{1}\right)$ and $\underline{w}(x)=0$ in $\left(x_{0}, z_{1}\right)$. Besides, since $w^{\prime} \geqslant 0$ in $(0, d)$, we deduce $w=0$ in $\left(x_{0}, d\right)$.

\subsection{The evolution problem}

Since the stationary solutions considered above are the long-term profiles for the time-dependent problem, the presence of a dead core in the stationary profile suggests that the non-stationary profile approaches the value 1 in that region. In fact, more is true: in this section we show that the timedependent profiles can attain the value 1, i.e. the dead core appears in finite time. Theorem 6.2, which gives sufficient conditions for the occurrence of dead cores, is proved by using local energy estimates, following the ideas of [2,7].

We first introduce some notation. Performing the change of unknown $v:=1-u$ in Problem $\mathrm{P}$ to remove the singularity from $u=1$ to $v=0$, we get

$$
\left\{\begin{array}{c}
v_{t}+(v q)_{x}-v_{x x}+f(x, 1-v)=0 \\
q_{x}+f(x, 1-v)=0 \\
\text { with } \\
\left.\begin{array}{c}
v(0, t)=1-u_{D}(t) \\
v_{x}(d, t)=q(d, t)=0
\end{array}\right\} \quad \text { in }(0, T) .
\end{array}\right.
$$

For any $t \in(0, T)$ we consider the set

$$
\mathcal{P}(t):=\left\{(x, \tau):\left|x-x_{0}\right|<R(\tau ; t), \quad \tau \in(t, T)\right\},
$$

with $R(\tau ; t):=(\tau-t)^{v}, 0<v<1$ to be fixed and $\alpha>0, x_{0} \in(0, d)$ such that

$$
R(T ; 0)<x_{0}<d-R(T ; 0),
$$




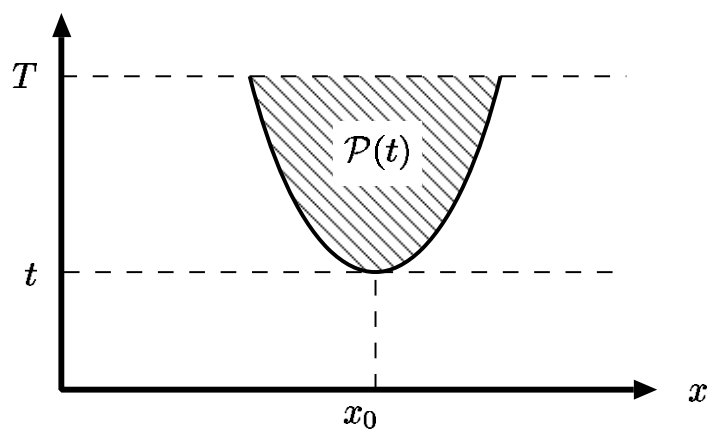

FIG. 2. The set $\mathcal{P}(t)$.

implying $\mathcal{P}(t) \subset Q_{T}$ for all $t \in(0, T)$ (see Fig. 2). For brevity, we shall write $\mathcal{P}$ instead of $\mathcal{P}(t)$. We decompose the boundary of $\mathcal{P}$ into final and lateral parts:

$$
\partial \mathcal{P}(t):=\partial_{f} \mathcal{P}(t) \cup \partial_{l} \mathcal{P}(t),
$$

with $\partial_{f} \mathcal{P}(t):=\{(x, T) \in \partial \mathcal{P}\}$ and $\partial_{l} \mathcal{P}(t):=\{(x, \tau) \in \partial \mathcal{P}: t<\tau<T\}$. Finally, we define the local energy functions

$$
E(t):=\int_{\mathcal{P}(t)}\left|v_{x}\right|^{2} \mathrm{~d} x \mathrm{~d} \tau \quad \text { and } \quad C(t):=\int_{\mathcal{P}(t)} v^{p+1} \mathrm{~d} x \mathrm{~d} \tau .
$$

Concerning function $f$ we assume, in addition to $(\mathrm{H})$, the existence of constants $k_{0}$ and $k_{1}$ such that

$$
0<k_{0} s^{p+1} \leqslant s f(\cdot, 1-s) \leqslant k_{1} s^{p+1} \quad \text { for } s \in[0,1]
$$

in $\mathcal{P}(t)$ for a.e. $t \in(0, T)$, with $p \in(0,1)$ and $k_{0}>k_{1} / 2$.

THEOREM 6.2 Assume (6.7). Then there exists a positive constant $M$ such that if $E(0)+C(0) \leqslant M$ then $v \equiv 0$ in $\mathcal{P}\left(t^{*}\right)$, for some $t^{*} \in(0, T)$.

\section{REMARK 6.2}

(i) If $f(x, u)=k(x)(1-u)^{p}$, with $k$ given by (2.14) then (6.7) is trivially satisfied in the region where $k \neq 0$.

(ii) Testing the first equation of (6.5) with $v$ and using the second equation of (6.5) leads to the following estimate:

$$
E(0)+C(0) \leqslant \int_{\Omega} v_{0}^{2}(x) \mathrm{d} x+\int_{0}^{T} v_{D}(t) v_{x}(0, t) \mathrm{d} t .
$$

In some situations, for instance when $v_{D}(t) \geqslant v_{0}(x)$ for $t \in(0, T)$ and $x \in(0, d)$, we have $v_{x}(0, t) \leqslant 0$ for $t \in(0, T)$, allowing us to obtain an estimate of $E(0)+C(0)$ only in terms of the initial datum. Notice that a typical data is $v_{D}=v_{0}=$ constant, for which the above condition is satisfied. 
Proof. The proof consists of three steps.

Step 1. Multiplying the first equation of (6.5) by $v$ and integrating in $\mathcal{P}$ gives

$$
\int_{\mathcal{P}}\left\{\frac{1}{2}\left(v^{2}\right)_{t}+\frac{1}{2}\left(\left(v^{2} q\right)_{x}+v^{2} q_{x}\right)+\left(\left|v_{x}\right|^{2}-\left(v v_{x}\right)_{x}\right)+v f(x, 1-v)\right\} \mathrm{d} x \mathrm{~d} \tau=0 .
$$

Using the divergence theorem, the second equation of (6.5) and (6.7) we find

$$
\begin{aligned}
\int_{\mathcal{P}}\left|v_{x}\right|^{2} \mathrm{~d} x \mathrm{~d} \tau \quad & +k_{0} \int_{\mathcal{P}} v^{p+1} \mathrm{~d} x \mathrm{~d} \tau \leqslant \int_{\partial_{l} \mathcal{P}} v v_{x} n_{x} \mathrm{~d} x \mathrm{~d} \tau \\
& -\frac{1}{2} \int_{\partial_{l} \mathcal{P}} v^{2}\left(n_{\tau}+q n_{x}\right) \mathrm{d} x \mathrm{~d} \tau+\frac{k_{1}}{2} \int_{\mathcal{P}} v^{p+2}
\end{aligned}
$$

with $\left(n_{x}, n_{\tau}\right)$ the unitary outward normal vector to $\mathcal{P}$, given by

$$
\left(n_{x}, n_{\tau}\right):= \begin{cases}(0,1) & \text { in } \partial_{f} \mathcal{P}, \\ \frac{\left((\tau-t)^{1-v},-v\right)}{\left(v^{2}+(\tau-t)^{2(1-v)}\right)^{1 / 2}} & \text { in } \partial_{l} \mathcal{P} .\end{cases}
$$

Using $v \leqslant 1, q \leqslant d k_{1}$ in $Q_{T}$ and $\left(n_{x}, n_{\tau}\right)$ unitary we obtain

$$
E(t)+\left(k_{0}-\frac{k_{1}}{2}\right) C(t) \leqslant \frac{1+d k_{1}}{2} \int_{t}^{T}\left[v^{2}\right] \mathrm{d} \tau+\int_{\partial_{l} \mathcal{P}}|v|\left|v_{x}\right| \mathrm{d} x \mathrm{~d} \tau,
$$

where we introduced the notation $[v]:=\left|v\left(x_{0}+R(\tau ; t), \tau\right)\right|+\left|v\left(x_{0}-R(\tau ; t), \tau\right)\right|$.

Step 2. Our aim is to estimate the right-hand side of (6.8) by means of the functions at the left-hand side and their derivatives. First notice that

$$
\frac{\mathrm{d} E}{\mathrm{~d} t}(t)=\int_{t}^{T}\left[\left|v_{x}\right|^{2}\right] \frac{\partial R}{\partial t}(\tau ; t) \mathrm{d} \tau,
$$

and therefore we can use Hölder's inequality to get

$$
\begin{aligned}
\int_{\partial \mathcal{P}}|v|\left|v_{x}\right| \mathrm{d} x \mathrm{~d} \tau & \leqslant\left(\int_{t}^{T}-\frac{\partial R}{\partial t}\left[\left|v_{x}\right|^{2}\right] \mathrm{d} \tau\right)^{1 / 2}\left(\int_{t}^{T}\left(-\frac{\partial R}{\partial t}\right)^{-1}\left[v^{2}\right] \mathrm{d} \tau\right)^{1 / 2} \\
& =I_{1}(t)\left(-\frac{\mathrm{d} E}{\mathrm{~d} t}(t)\right)^{1 / 2} \leqslant I_{1}(t)\left(-\frac{\mathrm{d}(E+C)}{\mathrm{d} t}(t)\right)^{1 / 2}
\end{aligned}
$$

with

$$
I_{1}(t):=\left(\int_{t}^{T}\left(-\frac{\partial R}{\partial t}\right)^{-1}\left[v^{2}\right] \mathrm{d} \tau\right)^{1 / 2} \quad \text { and } \quad I_{2}(t):=\int_{t}^{T}\left[v^{2}\right] \mathrm{d} \tau .
$$

To handle $I_{1}(t)$ and $I_{2}(t)$ of (6.8) we shall apply a simple version of an interpolation-trace inequality introduced in a more general setting in [7]. The proof is given in Section 6.2.1.

LEMMA 6.3 Let $\varphi \in H^{1}\left(x_{0}-\rho, x_{0}+\rho\right)$, for $x_{0} \in \mathbb{R}$ and a positive constant $\rho$. Then

$$
\left|\varphi\left(x_{0}-\rho\right)\right|+\left|\varphi\left(x_{0}+\rho\right)\right| \leqslant L_{0}\left(\left\|\varphi_{x}\right\|_{2}+\rho^{-\delta}\|\varphi\|_{p+1}\right)^{\gamma}\|\varphi\|_{r}^{1-\gamma},
$$


with $L_{0} \leqslant 16, r \in[1,2], p \geqslant 0$,

$$
\gamma:=\frac{2}{2+r} \quad \text { and } \quad \delta:=\frac{p+3}{2(p+1)} .
$$

Here we used the notation $\|\cdot\|_{s}:=\|\cdot\|_{L^{s}\left(x_{0}-\rho, x_{0}+\rho\right)}$.

We take $r<2$ and find, by applying Hölder's inequality with exponent $\theta:=\frac{1-p}{2-r}$

$$
\|\varphi\|_{r} \leqslant\|\varphi\|_{2}^{\frac{2}{r \theta^{\prime}}}\|\varphi\|_{p+1}^{\frac{p+1}{r \theta}} .
$$

Combining (6.10) and (6.12) with $\varphi(x):=v(x, \tau)$ and using $v \leqslant 1$ we get

$$
\left[v^{2}\right] \leqslant[v]^{2} \leqslant L_{0}^{2} m(R)\left(\left\|v_{x}\right\|_{2}^{2}+\|v\|_{p+1}^{p+1}\right)^{\gamma}\left|Q_{T}\right|^{\frac{2(1-\gamma)}{r \theta^{\prime}}}\|v\|_{p+1}^{\frac{2(1-\gamma)(p+1)}{r \theta}}
$$

with $m(R):=\max \left\{1, R^{-2 \delta \gamma}\right\}$. We then deduce from (6.13)

$$
I_{1} \leqslant L_{0}\left|Q_{T}\right|^{\frac{1-\gamma}{r \theta^{\prime}}}\left(\int_{t}^{T} m(R)\left(-\frac{\partial R}{\partial t}\right)^{-1}\left(\left\|v_{x}\right\|_{2}^{2}+\|v\|_{p+1}^{p+1}\right)^{\gamma+\frac{2(1-\gamma)}{r \theta}} \mathrm{d} \tau\right)^{1 / 2} .
$$

Due to the crucial assumption $p<1$, it is compatible to choose $r<2$ and $r \geqslant \frac{4}{3-p}$. Then we obtain that $\mu$ given by

$$
\mu^{-1}:=\gamma+\frac{2(1-\gamma)}{r \theta}
$$

satisfies $\mu \geqslant 1$. Using Hölder's inequality with exponent $\mu$ and substituting the explicit expression of $R$ we obtain from (6.14)

$$
I_{1} \leqslant \Lambda(t)(E(t)+C(t))^{\frac{\gamma}{2}+\frac{1-\gamma}{r \theta}},
$$

with

$$
\Lambda(t):=L_{0}\left|Q_{T}\right|^{\frac{1-\gamma}{r \theta^{\prime}}} \nu^{-1 / 2}\left(\int_{t}^{T}(\tau-t)^{\mu^{\prime}(1-\nu-2 \delta \nu \gamma)} \mathrm{d} \tau\right)^{1 / 2 \mu^{\prime}}
$$

Function $\Lambda$ is finite whenever we choose $v<\frac{\mu+1}{\mu(1+2 \delta)}$ which is always possible since the only restriction assumed on $v$ is $0<v<1$. Gathering (6.9) and (6.16) we get

$$
\int_{\partial \mathcal{P}}|u|\left|u_{x}\right| \mathrm{d} x \mathrm{~d} \tau \leqslant \Lambda(t)\left(-\frac{\mathrm{d}(E+C)}{\mathrm{d} t}(t)\right)^{1 / 2}(E(t)+C(t))^{\frac{\gamma}{2}+\frac{1-\gamma}{r}} .
$$

In a similar way, but choosing $r=2$ in (6.10), we get the following estimate:

$$
I_{2} \leqslant L_{0} \Gamma(t)(E(t)+C(t))
$$

with $\Gamma^{2}(t):=\int_{t}^{T}(\tau-t)^{-\delta v} \mathrm{~d} \tau<\infty$ if $v<1 / \delta$. 
Step 3. From (6.8), (6.14) and (6.18) we deduce

$$
c_{0}(E(t)+C(t)) \leqslant \Lambda(t)\left(-\frac{\mathrm{d}(E+C)}{\mathrm{d} t}(t)\right)^{1 / 2}(E(t)+C(t))^{\frac{\gamma}{2}+\frac{1-\gamma}{r}},
$$

with $c_{0} \leqslant k_{0}-\frac{k_{1}}{2}-\frac{1+d k_{1}}{2} L_{0} \Gamma(t)$. Notice that making $T-t$ small enough, say $T-t \leqslant \varepsilon$, we can ensure $c_{0}>0$. Making the assumption, to force a contradiction, that $E(t)+C(t)>0$ for all $t \in[0, T]$, we arrive at the inequality

$$
c_{0}^{2}(E(t)+C(t))^{2\left(1-\frac{\gamma}{2}-\frac{1-\gamma}{r}\right)} \leqslant-\Lambda(t)^{2} \frac{\mathrm{d}(E+C)}{\mathrm{d} t}(t) .
$$

Due again to $p<1$ we find $\sigma:=2\left(1-\frac{\gamma}{2}-\frac{1-\gamma}{r}\right)<1$. We assume $T>\varepsilon$ and restrict $t$ to take values on $(T-\varepsilon, T)$ (so $T-t \leqslant \varepsilon$ is fulfilled). Integrating (6.19) in $t \in\left(T-\varepsilon, t^{*}\right)$ with $t^{*} \in(T-\varepsilon, T)$ we obtain

$$
(E+C)^{1-\sigma}\left(t^{*}\right) \leqslant(E+C)^{1-\sigma}(T-\varepsilon)-(1-\sigma) c_{0}^{2} \int_{T-\varepsilon}^{t^{*}} \Lambda(t)^{-2} \mathrm{~d} t .
$$

Therefore, since $E+C$ is non-increasing we have that if the initial energy satisfies

$$
(E+C)^{1-\sigma}(0) \leqslant(1-\sigma) c_{0}^{2} \int_{T-\varepsilon}^{t^{*}} \Lambda(t)^{-2} \mathrm{~d} t=: M^{1-\sigma}
$$

then $E\left(t^{*}\right)+C\left(t^{*}\right)=0$ and therefore $v=0$ in $\mathcal{P}\left(t^{*}\right)$.

6.2.1 Appendix: an interpolation trace inequality. Lemma 6.3 is a particular case of a more general result obtained in [7] for any spatial dimension and a wider range of exponents. However, due to the space dimension, the proof is much simpler in our case, and all the constants appearing in it can be explicitly computed.

Proof of Lemma 6.3. We proceed in several steps:

Step 1 . We first consider $\varphi \in H^{1}(0,1)$. From the identity

$$
\varphi^{2}(x)-\varphi^{2}(0)=2 \int_{0}^{x} \varphi(x) \varphi_{x}(x) \mathrm{d} x
$$

and

$$
\varphi^{2}(0)=\int_{0}^{1} \varphi^{2}(x) \mathrm{d} x-2 \int_{0}^{1} \int_{0}^{x} \varphi(x) \varphi_{x}(x) \mathrm{d} x \leqslant\|\varphi\|_{2}^{2}+2\|\varphi\|_{2}\left\|\varphi_{x}\right\|_{2},
$$

we obtain

$$
\left[\varphi^{2}\right]:=\varphi^{2}(1)+\varphi^{2}(0) \leqslant 8\|\varphi\|_{2}\left(\|\varphi\|_{2}+\left\|\varphi_{x}\right\|_{2}\right)=8\|\varphi\|_{2}\|\varphi\|_{H^{1}(0,1)} .
$$

Step 2. If $\psi \in H^{1}(0,1)$ with $\psi(0)=0$ then from (6.20) and Hölder's inequality we get $\|\psi\|_{2} \leqslant$ $\sqrt{8}\left\|\psi_{x}\right\|_{2}$. Taking $\psi(x):=\varphi(x)-\varphi(0)$ we find

$$
\int_{0}^{1} \varphi^{2}+\varphi(0)^{2} \leqslant 8 \int_{0}^{1} \varphi_{x}^{2}+2 \varphi(0) \int_{0}^{1} \varphi \leqslant 8 \int_{0}^{1} \varphi_{x}^{2}+\varphi(0)^{2}+2\left(\int_{0}^{1} \varphi\right)^{2}
$$


and then $\|\varphi\|_{2} \leqslant \sqrt{8}\left(\left\|\varphi_{x}\right\|_{2}+\|\varphi\|_{1}\right)$, from where we deduce

$$
\|\varphi\|_{H^{1}(0,1)} \leqslant(\sqrt{8}+1)\left(\left\|\varphi_{x}\right\|_{2}+\|\varphi\|_{1}\right) .
$$

Step 3. We use Hölder's interpolation inequality:

$$
\|\varphi\|_{s} \leqslant\|\varphi\|_{l}^{\alpha}\|\varphi\|_{q}^{1-\alpha} \quad \text { with } \quad \frac{1}{s}=\frac{\alpha}{l}+\frac{1-\alpha}{q} \quad \text { and } \quad 1 \leqslant l \leqslant s \leqslant 2 \leqslant \infty .
$$

This inequality is true even if $l \in(0,1)$. Indeed, if we set $\varphi:=\psi^{m}$ with $m<\frac{1}{l}$ the above inequality reads $\|\varphi\|_{s m} \leqslant\|\varphi\|_{l m}\|\varphi\|_{q m}$, with $\frac{1}{s}=\frac{\alpha}{l}+\frac{1-\alpha}{q}$ and $l m<1$. Applying (6.23) to the function $\varphi^{2}$ with the parameters $s:=1, l=\alpha:=r / 2, q=\infty$ we get

$$
\|\varphi\|_{1}^{2} \leqslant\|\varphi\|_{r}^{r}\left\|\varphi^{2}\right\|_{\infty}^{1-r / 2} .
$$

By Sobolev's theorem

$$
\left\|\varphi^{2}\right\|_{\infty} \leqslant 2\left\|\varphi^{2}\right\|_{W^{1,1}(0,1)}=2\left(\|\varphi\|_{2}^{2}+\left\|\left(\varphi^{2}\right)_{x}\right\|_{1}\right),
$$

but $\left\|\left(\varphi^{2}\right)_{x}\right\|_{1} \leqslant 2\|\varphi\|_{2}\left\|\varphi_{x}\right\|_{2}$ and therefore

$$
\left\|\varphi^{2}\right\|_{\infty} \leqslant 4\|\varphi\|_{2}\left(\|\varphi\|_{2}+\left\|\varphi_{x}\right\|_{2}\right)=4\|\varphi\|_{2}\|\varphi\|_{H^{1}(0,1)} .
$$

We then obtain

$$
\|\varphi\|_{2}^{2} \leqslant\|\varphi\|_{r}^{r}\left(4\|\varphi\|_{2}\|\varphi\|_{H^{1}(0,1)}\right)^{1-r / 2}
$$

implying

$$
\|\varphi\|_{2} \leqslant 4^{\frac{2-r}{2+r}}\|\varphi\|_{r}^{\frac{2 r}{2+r}}\|\varphi\|_{H^{1}(0,1)}^{\frac{2-r}{2+r}} .
$$

Step 4. From (6.21) and (6.24) we get

$$
[\varphi] \leqslant\left[\varphi^{2}\right]^{1 / 2} \leqslant\left(4^{2+\frac{2-r}{2+r}}\|\varphi\|_{r}^{\frac{2 r}{2+r}}\|\varphi\|_{H^{1}(0,1)}^{\frac{2-r}{2+r}+1}\right)^{1 / 2}=2^{\frac{6+r}{2+r}}\|\varphi\|_{r}^{\frac{r}{2+r}}\|\varphi\|_{H^{1}(0,1)}^{\frac{2}{2+r}},
$$

and using (6.22) we obtain

$$
[\varphi] \leqslant 2^{\frac{10+r}{2+r}}\left(\left\|\varphi_{x}\right\|_{2}+\|\varphi\|_{1}\right)^{\frac{2}{2+r}}\|\varphi\|_{r}^{\frac{r}{2+r}} .
$$

Finally, since $\|\varphi\|_{1} \leqslant\|\varphi\|_{p+1}$ (recall that the measure of the domain is 1 ) we find

$$
[\varphi] \leqslant 2^{\frac{10+r}{2+r}}\left(\left\|\varphi_{x}\right\|_{2}+\|\varphi\|_{p+1}\right)^{\frac{2}{2+r}}\|\varphi\|_{r}^{\frac{r}{2+r}} .
$$

Notice that since $r \in[1,2]$ we have $2^{\frac{10+r}{2+r}} \leqslant 16$.

Step 5. Finally, we consider the change of unknown $y:=x_{0}-R+2 x R$ which maps the interval $(0,1)$ onto $\left(x_{0}-R, x_{0}+R\right)$. We obtain for any $q \geqslant 1$

$$
\|\psi\|_{L^{q}(0,1)}=\frac{1}{(2 R)^{q}}\|\psi\|_{L^{q}\left(x_{0}-R, x_{0}+R\right)}, \quad\|\psi\|_{H^{1}(0,1)}=\sqrt{2 R}\|\psi\|_{H^{1}\left(x_{0}-R, x_{0}+R\right)} .
$$

Therefore, from (6.25) we deduce

$$
[\varphi] \leqslant 2^{\frac{10+r}{2+r}}\left(\left\|\varphi_{x}\right\|_{2}+(2 R)^{-\frac{3+p}{2(p+1)}}\|\varphi\|_{p+1}\right)^{\frac{2}{2+r}}\|\varphi\|_{r}^{\frac{r}{2+r}}
$$

for any $\varphi \in H^{1}\left(x_{0}-R, x_{0}+R\right)$. 


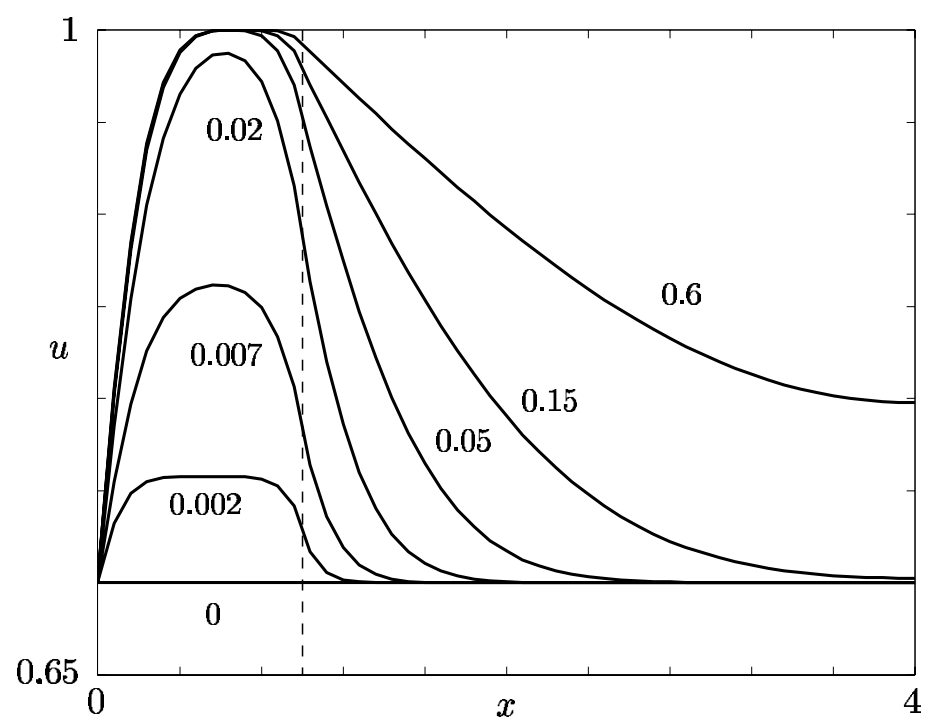

FIG. 3. Evolution of dimensionless salinity $u$ for root distribution $k(z)$ given by (2.14), and for parameter values $p=0.5$, $k_{0}=15, d=4$, and $u_{0}=0.7$. Numbers in the figure are values of $t$.

\section{Numerical examples}

For the numerical solution of Problem $\mathrm{P}$ we considered the equivalent non-local formulation

$$
\begin{cases}u_{t}+\left(u \int_{x}^{d} f(\cdot, u)\right)_{x}-u_{x x}=0 & \text { in } Q_{T}, \\ u(0, t)=u_{D}(t), \quad u_{x}(d, t)=0 & \text { for } 0<t<T, \\ u(x, 0)=u_{0}(x) & \text { for } 0<x<d,\end{cases}
$$

which is obtained after the integration in $(x, d)$ of the second equation of Problem $\mathrm{P}$. To compute approximate solutions we employed an explicit upwind finite difference scheme with 51 equidistributed spatial nodes in the region $0 \leqslant x \leqslant d=4$, and with fixed time increment steps.

We calculated numerical solutions for the evolution of the $u(x, t)$ profile when $u_{D}=u_{0}=0.7$ and $f$ takes the form (2.13), with a non-uniform root distribution, $k$, given by (2.14) with $k_{0}=15$.

We investigated the effects of linear $(p=1)$ and strong $(0<p<1)$ absorption on the formation of dead cores. Figs 3 and 4 show several time slices of $u$ for $p=0.5$ and $p=1$, respectively. It is clear from the pictures that in the superlinear case a dead core arises in finite time, meanwhile it never occurs in the linear case. Remark that the region $1<x<d$ salinizes slowly by diffusion of salt down from the root zone.

Note that in the case $p<1$ the results of Section 4 do not guarantee uniqueness of the solutions calculated here. However, as remarked before, we believe that the hypotheses of Theorem 4.2 are unnecessarily restrictive, and that uniqueness in fact holds in a suitably general sense. This should also include the cases studied here numerically. 


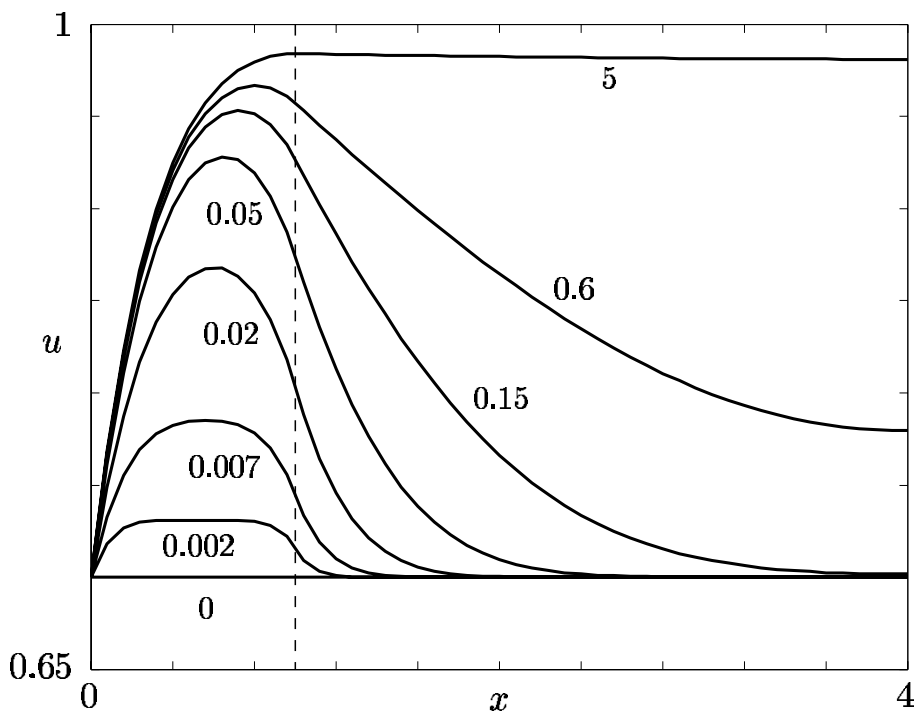

FIG. 4. As Fig. 3, but for $p=1$.

\section{Discussion and conclusion}

In the preceding sections we have analysed a problem that arises in the study of soil salinization. The root systems of mangroves extract water from the soil, leaving most of the dissolved salt behind. Since the sea water that enters the soil to replace the extracted water is saline, the salt concentration $c$ in the root zone increases. One of the aims of this paper was to investigate the possibility that this mechanism can (locally) bring the water uptake to a complete standstill. In order to study this question, a system of equations was formulated that govern the transport of both salt and water in a general time-dependent setting. We introduced a critical salt concentration $c_{c}$, above which the root system is unable to extract water.

The stationary version of this problem is relatively simple in nature, and allows for a detailed analysis. We used a phase plane approach to investigate solutions of this problem, and discussed some qualitative properties. We showed that if the water uptake function is proportional (at least locally, around $\left.c=c_{c}\right)$ to $\left(1-c / c_{c}\right)^{p}$, then the character of the solutions depends critically on $p$. If $p \geqslant 1$, then the stationary salt profile shows an increased level of salt, but this level remains below $c_{c}$, and therefore water continues to be absorbed. On the other hand, if $0<p<1$, then under suitable conditions a 'dead core' forms, in which the critical concentration is attained, and therefore no water is extracted.

The time-dependent problem exhibits a coupling of the mass-balance and the fluid-balance equations via convective terms. The theory of such systems is relatively underdeveloped, and no general well-posedness results in the literature are known to us. In this paper we have proved a general existence theorem, as well as a uniqueness/comparison result. However, the latter requires an a priori restriction on the solution, and although this restriction appears purely technical, other problems are known in which similar restrictions appear [6], and the question of the optimal hypotheses for a uniqueness result remains open. The comparison principle only holds for this 
system of equations when one of the solutions is monotone; this condition can not, in general, be relaxed.

The stationary solutions are natural candidates for the long-term behaviour of the timedependent solutions, and in Section 5 we show that this suggestion is justified. However, the comparison principle is necessary in the current formulation of the proof of this result, and therefore the theorem only applies to a certain class of initial data. This restriction again appears to be purely technical, and this is also borne out by numerical simulations.

The behaviour of the root water uptake function near the critical salt concentration is crucial in determining the appearance of dead cores, not only for the stationary solutions but also for timedependent ones. In Section 6 we employ a method based on local energy estimates to show that if $0<p<1$, the salt concentration may attain the critical value $c_{c}$ after finite time.

\section{Acknowledgement}

GG appreciates the encouraging atmosphere found in his stay at CWI during 1997. All three authors wish to acknowledge the contribution of John H. Knight, and wish to express their gratitude to him for bringing this problem to their attention.

\section{REFERENCES}

1. Amann, H. Gewöhnliche Differentialgleichungen. Berlin (1983).

2. Antontsev, S. N., DíAZ, J. I., \& Shmarev, S. I. The support shrinking properties for local solutions of quasilinear parabolic equations with strong adsorption terms. Ann. Fac. Sciences de Toulouse 4, (1995) 5-30.

3. Arino, O., Gauthier, S., \& Penot, J. P. A fixed point theorem for sequentially continuous mappings with application to ordinary differential equations. Funkcial Ekvac. 24, (1987) 273-279.

4. BeAR, J. Dynamics of Fluids in Porous Media. American Elsevier, New York (1972).

5. DíAZ, J. I. Nonlinear Partial Differential Equations and Free Boundaries. Volume I: Elliptic equations, Research notes in mathematics. Pitman, London (1985).

6. DíAZ, J. I., GALIANO, G., \& JÜNGEL, A. Space localization and uniqueness of solutions of a quasilinear parabolic system arising in semiconductor theory. C. R. Acad. Sci. Paris I 325, (1997) 267-272.

7. DÍAZ, J. I. \& VERON, L. Local vanishing properties of solutions of elliptic and parabolic quasilinear equations. Trans. Am. Math. Soc. 290, (1985) 787-814.

8. VAN DUiJn, C. J. \& KNABnER, P. Travelling waves in transport of reactive solutes trough porous media: adsorption and binary ion exchange-Part 2. Transport in Porous Media 8, (1992) 199-225.

9. Hutchings, P. \& SAenger, P. Ecology of Mangroves. University of Queensland Press, Queensland (1987).

10. Ladyzenskaya, O. A., Solonnikov, V. A., \& Ural'Ceva, N. N. Quasilinear Equations of Parabolic Type, Translations of Mathematical Monographs, 23. American Mathematical Society, Providence (1968).

11. Lin, G. \& Stenberg, L. DA S. L. Utilization of surface water by red mangrove (Rhizophora Mangle L.): an isotopic study. Bull. Mar. Sci. 54, (1994) 94-102.

12. Lions, J. L. Quelques Méthodes de Résolution des Problèmes aux Limites Non Linéaires. Dunod, Gauthiers-Villars, Paris (1969).

13. Passioura, J. B., Ball, M. C., \& Knight, J. H. Mangroves may salinize the soil and in so doing limit their transpiration rate. Funct. Ecol. 6, (1992) 476-481.

14. Simon, J. Compact sets in the space $L^{p}(0, T ; B)$. Ann. Math. Pures et Appl. 146, (1987) 65-96. 